
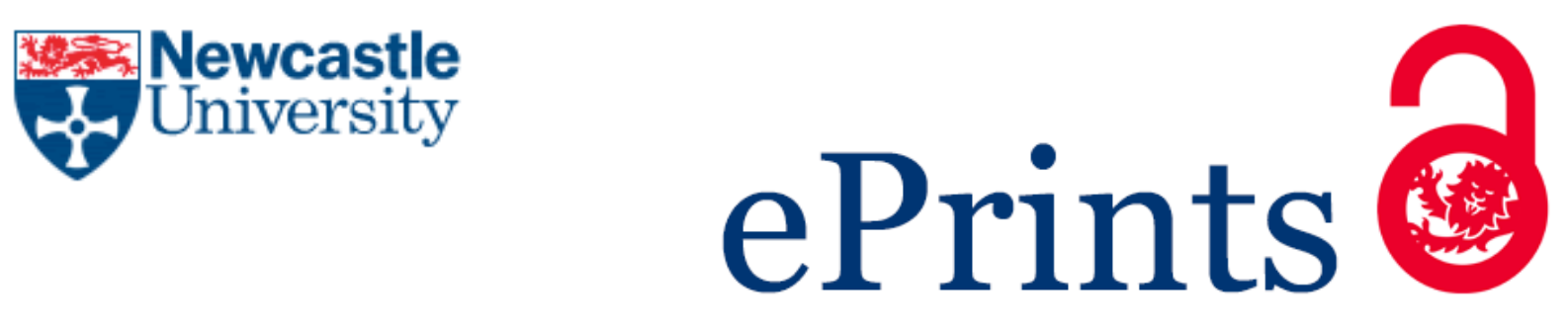

Van Dijck P, Brown NA, Goldman GH, Rutherford J, Xue C, Van Zeebroeck G.

Nutrient sensing at the plasma membrane of fungal cells.

Microbiology Spectrum 2017, 5(2)

\title{
Copyright:
}

(C) 2017 American Society for Microbiology.

DOI link to article:

https://doi.org/10.1128/microbiolspec.FUNK-0031-2016

Date deposited:

$15 / 06 / 2017$

Embargo release date:

10 September 2017 
Section 4: how fungi sense their environment

\section{Nutrient sensing at the plasma membrane of fungal cells}

Patrick Van Dijck ${ }^{1,2}$, Neil Andrew Brown ${ }^{3}$, Gustavo H. Goldman ${ }^{4}$, Julian Rutherford ${ }^{5}$, Chaoyang Xue ${ }^{6}$, Griet Van Zeebroeck ${ }^{1,2}$

${ }^{1}$ VIB Department of Molecular Microbiology, KU Leuven, Flanders, Belgium. ${ }^{2}$ Laboratory of Molecular Cell Biology, Institute of Botany and Microbiology, KU Leuven, B-3001 Leuven, Belgium

${ }^{3}$ Plant Biology and Crop Science, Rothamsted Research, Harpenden, AL5 2JQ, UK

${ }^{4}$ Faculdade de Ciências Farmacêuticas de Ribeirão Preto, Universidade de São Paulo, Ribeirão Preto, São Paulo, Brazil

${ }^{5}$ Institute for Cell and Molecular Biosciences, Medical School, Newcastle University Catherine Cookson Building, Framlington Place, Newcastle upon Tyne, NE2 4HH, UK

${ }^{6}$ Public Health Research Institute, Department of Microbiology, Biochemistry and Molecular Genetics, Rutgers Biomedical and Health Sciences, Newark, New Jersey 07103, USA

\section{Introduction}

In order to respond to the changing environment, cells must be able to sense external conditions. This is important for many processes including growth, mating, the expression of virulence factors and several other regulatory effects. Nutrient sensing at the plasma membrane is mediated by different classes of membrane proteins that activate downstream signaling pathways: non-transporting receptors, transceptors, classical and non-classical G-protein coupled receptors and the newly defined extracellular mucin receptors. Non-transporting receptors have the same structure as transport proteins, but have lost the capacity to transport while gaining a receptor function. Transceptors are transporters that also function as a receptor, as they can rapidly activate downstream signaling pathways. In this review we focus on these four types of fungal membrane proteins. We mainly discuss the sensing mechanisms relating to sugars, ammonium and amino acids. Mechanisms for other nutrients, such as phosphate, sulfate, are discussed briefly. As the model yeast Saccharomyces cerevisiae has been the most studied, especially regarding these 
nutrient sensing systems, each subsection will commence with what is known in this species.

\section{Abbreviations}

PKA: protein kinase A; ITR: inositol transporter; CAZymes: carbohydrate-active enzymes; MFS: major facilitator superfamily; AAP: amino acid permease; GPCR: Gprotein coupled receptor; NCR: nitrogen catabolite repression; RGS: repressor of Gprotein signaling; SCAM: substituted cysteine accessibility method; AC: adenylate cyclase.

\section{Non-transporting receptors sensing glucose}

\subsection{Glucose sensing by Snf3 and Rgt2 in S. cerevisiae.}

The uptake of glucose, the preferred carbon source of $S$. cerevisiae, is mediated by 17 closely related hexose transporters (1). Their expression is coordinated according to the availability of extracellular glucose, which is sensed by the two glucose receptors, Snf3 and Rgt2 (2) (see 5.1 and Figure 1). Snf3 and Rgt2 sense the internal-external ratio of glucose in order to adjust glucose uptake (3), which is also influenced by intracellular glucose metabolism (glycolysis) (4). Although these receptors are structurally similar to hexose transporters, they cannot transport glucose and contain unusual long C-terminal tails that are important for glucose sensing (5).

In the absence of glucose, the Rgt1 transcriptional repressor is found in the nucleus, interacting with the co-repressors Mth1-Std1 (6), thereby recruiting the general corepressor complex Ssn6-Tup1 to the promoters of the different HXT genes, blocking their transcription (7). Snf1 dependent phosphorylation of Rgt1 triggers its repressor activity as well as its propensity to bind DNA (8). In addition, Snf1 also affects $H X T$ expression via repression of the carbon catabolite repressors, Mig1/2, which in turn repress the expression of STD1, MTH1 and SNF3 genes (9). Glucose detection by Snf3-Rgt2 recruits the corepressors Mth1-Std1 to the plasma membrane where they are subsequently phosphorylated by type I casein kinases Yck1/2 (10). Upon phosphorylation, Mth1-Std1 are targeted for SCF E3 ligase Grr1mediated ubiquitination and proteasomal degradation (11). Collectively, these events lead to a protein kinase A (PKA) dependent hyperphosphorylation of Rgt1, which induces the dissociation of Ssn6-Tup1 from the DNA, resulting in the derepression of the $H X T$ genes $(12,13)$. 
Homologs of the ScSnf3-ScRgt2 pathway have been identified in other yeasts (Figure 1). In Kluyveromyces lactis, this is the Rag4 sensor (14), which regulates the expression of the glucose transporter gene RAG1 (15). Also the downstream regulatory pathway is similar to that described for $S$. cerevisiae (16-18). In the methylotrophic yeast Hansenula polymorpha, the HpHxs1 is nonfunctional as a glucose transporter, but is responsible for the glucose-dependent induction of the functional transporter, $\mathrm{HpHxt1}$ (19). The C-terminus of this receptor is essential for its function and the mutation of one conserved residue (R204K) converts it into a constitutively signaling form.

\subsection{Carbon sensing in Candida albicans and Cryptococcus neoformans}

C. albicans has over 20 hexose transporter homologs (20). Among them, Hgt4, an orthologue of the S. cerevisiae glucose receptors Snf3 and Rgt2, has been identified as a high affinity glucose sensor (21) (Figure 1). Hgt4 shares sequence and structure similarity with other hexose transporters, with the exception of a long Cterminal tail containing 254 aa, similar to Snf3 and Rgt2. Hgt4 is required for glucose induction of the hexose transporter genes HGT12, HXT10, and HGT7. Mutagenesis demonstrated that the hgt $4 \Delta$ mutant is defective in growth on fermentable sugars, such as fructose, mannose, and glucose. The hgt $4 \Delta$ mutants are hypofilamentous, indicating that Hgt4 affects the yeast-to-hyphal morphological switch. Interestingly, Hgt4 is also required for fungal virulence as the mutant showed attenuated virulence in a murine model for systemic infection. These findings demonstrate that $C$. albicans cells need to sense and regulate sugar levels for fungal filamentation during infection. The downstream regulatory mechanism of glucose repression in $C$. albicans is conserved with its counterpart in $S$. cerevisiae $(12,22)$. However, it remains unclear if $\mathrm{Hgt} 4$ has glucose uptake activity or not.

Also in C. neoformans, glucose sensing and utilization is critical for its development and virulence. Besides being utilized as a preferred energy source for cell growth, glucose is required for capsule production both as a substrate and a signaling molecule. Glucose induces capsule enlargement through the Gpa1-cAMPPKA signal transduction pathway, which plays a central role in fungal virulence (see 5.2) (23). Despite its biological significance, glucose sensing is less well understood in C. neoformans, compared to $S$. cerevisiae and C. albicans. There are $\sim 50$ transporters that share high sequence similarity with hexose transporters (24). Although some of them may transport sugars other than glucose, how this large family of hexose transporters is regulated remains unknown. Among these hexose transporter homologs, Hxs1 and Hxs2 share the highest sequence identity with 
ScRgt2/ScSnf3 glucose receptors, but neither of them have a long C-terminal tail (24). Hence, it is possible that the $C$. neoformans glucose sensing system is different from S. cerevisiae and $C$. albicans. These two proteins will be discussed in the transceptor section as evidence suggests that they may have both transport and regulatory functions (Figure 1).

Inositol is a small carbohydrate molecule that functions as an essential structural and signaling molecule in eukaryotes, including fungi (25). In $C$. neoformans, inositol can be used as a sole carbon source (26). Inositol can also stimulate Cryptococcus mating (27). As one of the most abundant metabolites in the mammalian brain (28), inositol utilization is required for $C$. neoformans virulence in murine infection models by promoting brain infection (29-32). Therefore, C. neoformans is likely to utilize the abundant inositol available inside the brain for its pathogenesis. Inositol can also stimulate $C$. neoformans capsule growth, which may contribute to its role in fungal virulence. The cryptococcal genome reflects the evolutionary adaptations associated with the expanded role of inositol in this organism. In particular, C. neoformans contains an unusually large number of inositol transporters (ITRs) that consists of more than ten members $(27,29)$. Functional analysis of the ITR gene family demonstrated that two members (Itr1a and Itr3c) have high inositol uptake activity, while the function of the other members remains undefined. Among them, Itr1 was suggested as an inositol transceptor as it does not show uptake activity in the yeast heterologous system, it regulates the expression of ITR genes and the itr1 single mutant exhibits a defect in mating, hyphal production and sporulation $(29,32)$. Therefore, transceptors for inositol may exist in $C$. neoformans.

\subsection{Carbon sensing in filamentous fungi}

The mechanisms by which filamentous fungi sense saccharides in the environment are beginning to emerge. It is becoming increasingly clear that due to the metabolic plasticity of filamentous fungi and their ability to utilize numerous nutrient sources, sensory mechanisms and pathways are far more complex than that in yeasts. This knowledge has been fundamental to the industrial application of filamentous fungi in the production of second generation biofuels and green chemistries. Lignocellulolytic filamentous fungi, such as Trichoderma reesei, Aspergillus nidulans and Neurospora crassa, secrete an array of carbohydrate-active enzymes (CAZymes) that deconstruct and utilize lignocellulose. Primarily the activity of a few scavenging fungal enzymes releases potent saccharide inducers, such as cellobiose, from the plant biomass, which induces the secretion of hundreds of 
CAZymes by the fungus, promoting lignocellulose deconstruction (33). However, the detection of simple saccharides, such as glucose, xylose or an abundance of cellobiose, results in CreA/Cre1-mediated carbon catabolite repression (33). Therefore, distinct mechanisms for sensing plant derived saccharides exist to enable the efficient regulation of fungal metabolism and alternative carbon usage.

In contrast to the aforementioned yeasts, homologs of the Snf3 and Rgt2 glucose receptors have not been functionally defined in filamentous fungi. In $T$. reesei a putative MFS sugar transporters Crt1 was required for full cellulase activity and growth on cellulose or lactose (34). The absence of Crt1 did not compromise cellobiose or sophorose uptake, implying that the function of Crt1 in cellulase induction did not rely on its transporting activity. However, in N. crassa cellobiose promotes cellulase production independent of glucosidase activity, indicating that cellobiose itself is the inducer for the cellulolytic regulon (35), suggesting that cellobiose detection may occur prior to internalization. However, only a few cases of non-transporting (putative) receptors have been reported in filamentous fungi. In addition, a novel cellodextrin transporter-like protein, Clp1, also forms a critical component in the regulation of cellulase production (36). However, Clp1 did not import cellodextrins, and actually suppressed the transcription of cellulases and other cellodextrin transporters in response to cellobiose or cellulose (36). Therefore, despite the knowledge of non-transporting receptors in filamentous fungi being elusive, they appear to exist and can function either as inducers or repressors of the lignocellulolytic machinery.

\section{2: Non-transporting receptors sensing nitrogen (amino acids, ammonium, other sources)}

Nitrogen is essential for the synthesis of many cellular components (amino acids, nucleotides, ...). Therefore cells developed a whole series of systems to sense and take up these nutrients. Apart from plasma membrane localized systems, discussed in this chapter, also the target of rapamycin complex 1 (TORC1) is a major growth regulator in response to nitrogen quality and quantity. This is described in detail in the chapter on TOR signaling in this book section.

\subsection{Amino acid sensing by Ssy1 in S. cerevisiae}

In $S$. cerevisiae, amino acids are detected at the plasma membrane by a multiprotein complex composed of Ssy1, Ptr3 and Ssy5 (SPS) (37) (Figure 2). This results in induced expression of amino acid transporter genes as well as other 
nitrogen metabolizing enzymes (38). Ssy1 is a unique member of the amino acid permease (AAP) family and is the receptor of the SPS (Ssy1-Ptr3-Ssy5) complex (39). Although Ssy1 does not transport amino acids, it does undergo a conformational change upon binding of its ligand (40). Direct binding of the amino acid to Ssy1 was proven by the identification of hyper- and hyporesponsive mutants $(41,42)$. The extremely long Ssy1 $\mathrm{N}$-terminal tail is highly conserved in other yeast species, and it is required for its function as a sensor-signal transducer (43).

Ptr3, the least understood component of the complex, is induced by Yck1/2dependent phosphorylation (44). Recent results indicate a key role for Ptr3 as an adaptor protein, linking the amino acid induced conformational change of Ssy1 to Yck1/2-Ssy5 access (45). Ptr3 multimerization, a well-established concept for Gprotein coupled receptors (GPCRs) as well as receptor tyrosine kinases (46, 47), occurs via the WD40-like domains, and is essential for downstream activation.

The chymotrypsin-like protease Ssy5 is synthesized as two domains, a catalytic domain and an inhibitory prodomain. Autocatalytic cleavage occurs during synthesis, however the parts remain associated forming an inactive complex (48). In the presence of amino acids, the prodomain is released, leading to activation. The release of Ssy5 requires phosphorylation by type I casein kinases Yck1/2 followed by ubiquitination by SCFGrr1 $(49,50)$. Ubiquitination leads to proteasomal degradation of the prodomain, yet the direct involvement of the $26 \mathrm{~S}$ proteasome has been questioned (50). The Ssy5 prodomain contains a phosphodegron, which may represent the site of Yck1/2 recognition (51). This sequence was also found in Mth1 and Std1, effector components of the Snf3/Rgt2 glucose-sensing pathway that also requires Yck1/2 and SCFGrr1 (52).

The amino acid induced receptor activated proteolysis (RAP) of Ssy5 results in endoproteolytic removal of the N-terminal tail of the transcription factors Stp1/2, which are synthesized as latent cytoplasmatic precursors (53). The processed forms of Stp $1 / 2$ are translocated into the nucleus where they bind to, and induce the expression of, their target genes. Although homologous, Stp1/2 are functionally divergent, possessing a different $\mathrm{N}$-terminal tail, and also showing differential degradation and distinct cellular localization, providing a mechanistic explanation for Stp2 being the primary activator $(54,55)$.

Several control levels to maintain the derepressed state of signaling in the absence of inducing amino acids exist. Firstly, Ssy5 activity is controlled by its Rts1/PP2A dependent dephosphorylation, which antagonizes the Yck1/2-dependent phosphorylation of the prodomain, and thereby mute Ssy 5 activity in the absence of amino acids $(44,45,56)$. Second, the Asi1-3 inner nuclear membrane proteins 
restrict entrance of full-length Stp $1 / 2$ in the nucleus due to its $\mathrm{N}$-terminal region (57, 58). A third level of regulation is the targeting of unprocessed Stp1 for degradation, thereby diminishing its nuclear abundance (59). An additional level of Stp1 regulation occurs by TOR, inactivation of TOR by rapamycin results in the degradation of Stp1 in the nucleus, which is dependent on the phosphatase 2A (PP2A)-like phosphatase, Sit4 (60). All these control systems show that the cells have invested a lot in order to have activation of the pathway only in conditions where this is relevant. More information on TOR signaling is presented in Chapter XXX

\subsection{Amino acid sensing in C. albicans and C. neoformans}

The transcriptional regulation of AAP genes by the presence of their substrates in C. albicans occurs in a similar manner to that of $S$. cerevisiae (61). The Candida genome encodes homologs of all characterized components of the SPS complex (62). CaCsy1, the ScSsy1 homolog, similarly acts as the primary receptor (Figure 2). Despite the high degree of homology, the $\mathrm{N}$-terminus of both proteins is very divergent, potentially contributing to their differences in specificity (61). C. albicans cells lacking CaCsy1 show altered morphology and hyphal formation, indicating a role in amino acid- (serum-) induced hyphal morphogenesis.

Similar to $S$. cerevisiae, the sensor complex induces proteolytic processing of two latent transcription factors, CaStp1/2, upon amino acid detection (63). Both proteins are activated upon amino acid addition, to differentially activate two modes of nitrogen acquisition: CaStp2 activates genes required for amino acid uptake, while CaStp1 induced genes involved in extracellular protein degradation and peptide uptake. In addition, the levels of CaStp1 itself are also amino acid regulated, indicating that Candida cells preferentially use amino acids (63). Recent results indicate that the control of STP1 expression is mediated by the two GATA transcription factors CaGln3 and CaGat1 (64). Using the insect host, Drosophila, it was shown that a functional SPS sensor and proper Stp1 gene control are required for host protein catabolism and utilization, while being essential for full virulence. CaStp2, on the other hand, is dispensable for virulence (65).

Recently, eight global affinity amino acid permeases and two methionine permease homologs have been identified in C. neoformans (66). From this low number of permeases, it is apparent that this pathogen is more dependent on amino acid biosynthesis than on uptake. Transcriptional profiling revealed that these genes are subjected to regulatory mechanisms known to respond to the nutritional status in other fungi, such as nitrogen and carbon catabolite repression, SPS sensing as well as general amino acid control. Inhibitors of these pathways caused in vitro growth 
arrest. Despite the presence of ScSSY1 and ScSTP2 homologs, an SPS sensing pathway as in $S$. cerevisiae or $C$. albicans has never been described in $C$. neoformans.

To date, non-transporting amino acid receptors have not yet been described in filamentous fungi while non-transporting ammonium sensors have not been described in any fungal species.

\section{Transceptors (transporting receptors) sensing carbon sources}

\subsection{Transceptor-mediated carbon sensing in S. cerevisiae and other yeasts}

In $S$. cerevisiae, none of the hexose carriers has been directly implicated in activating/repressing downstream signaling pathways. Glucose repression appears to be independent of plasma membrane transceptors (67). This is different from the situation in mammalian cells where transporters such as GLUT1, GLUT2 and GLUT4 have been shown to activate downstream signaling pathways (68-71).

\subsection{Transceptor-mediated carbon sensing in C. albicans and C. neoformans}

The presence of carbon-sensing transceptors in $C$. albicans or $C$. neoformans has not been clearly demonstrated. In C. albicans, Hgt4 and Hgt12 have been reported to be transceptors which regulate glucose sensing and uptake (Figure 1). Whether Hgt4 has glucose uptake activity remains untested, but Hgt12 seems to have glucose transport activity, potentially indicating that this may be a bonafide transceptor $(21,72,73)$. Hgt12 is specifically expressed during macrophage infection, probably as a response to the absence of glucose in the phagolysosome (72). In C. neoformans, Hxs1 has high sequence identity with ScSnf3/ScRgt2, yet it still shows glucose uptake activity (24). Mutagenesis showed that Hxs1 is required for efficient glucose uptake and fungal growth under low glucose conditions. Hxs 1 is not required for regulating the expression of hexose transporter candidates and heterologous expression of HXS1 does not complement the S. cerevisiae snf3 $\Delta$ rgt2 $\Delta$ phenotype. Therefore, whether Hxs1 also functions as a glucose sensor remains to be defined. Importantly, however, Hxs1 is required for fungal virulence in a murine infection model of cryptococcosis. Whether Hxs2 could function as a glucose sensor remains unclear. Interestingly, Hxs1 and Hxs2 do not have the long C-terminal tail that has been shown to be essential for sensory function of their orthologs in S. cerevisiae and C. albicans. Similarly, in the methylotrophic yeast Hansenula polymorpha, the hexose transporter homolog Gcr1 also lacks the typical C-terminus, but its regulatory role, along with its involvement in hexose transport, has 
been demonstrated (74). Therefore, a long C-terminal tail may not be essential for glucose sensing in some fungal glucose transceptors.

\subsection{Transceptor-mediated carbon sensing in filamentous fungi}

In filamentous fungi, transceptors are proposed to perform roles in the detection and uptake of polysaccharides, which in turn influences how these fungi interact with their environment. A dual role in signaling and in sugar transport has been shown for $N$. crassa Rco3, lacking the unique C-terminus, although it is not fully clear whether this protein has an active transport capacity $(14,75)$ (Figure 1). Similarly, Hxt4 from the hemibiotrophic maize pathogen Collectrichum graminicola which has very low similarity to the ScHxt, ScSnf3 or ScRgt2 receptors in $S$. cerevisiae, was shown to be a sensor of nutritional status (76). Recently, Hxt1 from Ustilago maydis, a ubiquitous pest of corn, was characterized as a high-affinity hexose transporter that also functions as a receptor, which is required for full virulence (77). First, overexpression of a mutant (R164K), resulting in constitutive signaling in Snf3/Rgt2, leads to downregulation of hexose transport under glucose deprivation and complete loss of virulence. Second, heterologous expression of highaffinity transporters C. graminicola HXT3 and Arabidopsis thaliana STP1 only complement the growth defect of an $h x t 1 \Delta$ strain and not its decreased virulence. How exactly the signal is transduced downstream is not yet elucidated. However, $U$. maydis possesses a homolog of the membrane-bound casein kinase I (Yck1/2), but no homologs of all the other downstream components of the ScSnf3/ScRgt2 pathway have been identified.

Transporters of cellodextrins, the breakdown product of cellulose, have been identified and shown to be fundamental to the regulation of cellulase production. Two cellodextrin transporters, Cdt1 and Cdt2, from $N$. crassa, were shown to contribute to cellulose sensing and mutants lacking both transporters were unable to induce cellulases in response to cellulose (78). Insertion of these cellodextrin transporters into $S$. cerevisiae along with a $N$. crassa $\beta$-glucosidase enabled cellobiose fermentation $(79,80)$, demonstrating their transporter activity. However, the impairment of cellobiose transport, by introducing point mutations into CDT-1 or CDT-2, did not greatly impact on cellulase induction in response to cellobiose, suggesting that the cellodextrin transporters act as transceptors with dual functions in the transport and the detection of cellobiose (78). In T. reesei, Stp1, an MFS sugar transporter, capable of transporting cellobiose was identified. However, its absence enhanced cellulase production, demonstrating how Stp1 repressed cellulase production (34). Hence, these studies have provided novel insights into the detection 
of cellulose by filamentous fungi and how cellobiose transceptors can regulate the lignocellulolytic enzyme production.

\section{Transceptors sensing nitrogen (amino acids, ammonium, other sources)}

\subsection{Transceptor-mediated amino acid sensing in S. cerevisiae}

In S. cerevisiae, the uptake of amino acids involves several general as well as specific transporters, all belonging to the AAP family (81). One of the general permeases is Gap1, a proton symporter catalyzing transport of all L-amino acids including L-citrulline, 4-amino butyric acid, some D-amino acids and toxic analogs as well as polyamines $(82,83)$ (Figure 2). The expression as well as the activity of Gap1 are tightly regulated by both the quality and quantity of the nitrogen sources available. Transcriptional regulation occurs via the nitrogen catabolite repression pathway, by the transcriptional activators, Gln3 and Gat1, and inhibitors, Dal80 and Deh1 $(84,85)$. Fine-tuning of the amount of plasma membrane localized molecules according to nitrogen availability is regulated by the coordinated action of the Npr1 kinase, the $\alpha$-arrestins Aly $1 / 2$ as well as the ubiquitin ligase adaptors Bul1/2 leading to ubiquitination of Gap1 by the ubiquitin-ligase Rsp5, followed by endocytosis and degradation in the vacuole (86-89). Some amino acid analogs however trigger oligoubiquitination without endocytosis, indicating that an additional modification is required to initiate the internalization process (90). An additional level of regulation at the plasma membrane is reversible inactivation, due to a conformation change, upon binding of amino acids (91).

Upon studying the amino acid induced activation of the PKA pathway it was found that the well-known transporter Gap1 has an additional role as an amino acid receptor (92). The involvement of metabolism and intracellular sensing has been excluded in order to prove the direct receptor function. High L-citrulline concentrations do not trigger signaling in a gap1 $1 \Delta$ strain, despite its transport by other carriers. The involvement of metabolism was excluded as non-metabolizable D-amino acids trigger the same activation as their metabolizable L-counterparts. Moreover, deletion of the first enzyme required for L-citrulline catabolism does not abolish L-citrulline induced activation. Direct binding of the agonists as well as identification of two residues, $\operatorname{Ser}^{388}$ and $\mathrm{Val}^{389}$, exposed with their site chain in the binding site, was obtained by substituted cysteine accessibility method (SCAM) analysis of the eighth transmembrane domain (93). An additional strong argument that transport through the transceptor is not required to trigger signaling was the identification of non-transported signaling agonists. A recent study showed that for 
both signaling and endocytosis, different substrates elicit specific conformational changes upon movement through the passageway, indicating that all these events (transport, signaling or endocytosis) can occur independently of one another (90). Despite indications for involvement of certain proteins in the downstream signaling, such as the PKA complex, the Sch9 protein kinase and the S. cerevisiae PDK1 orthologues (Pkh1-3), the exact mechanism by which the Gap1 transceptor relays the signal downstream, remains unclear $(94,95)$. For example, a basal level of cAMP is required, but there is no transient increase in cAMP upon amino acid activation (96).

\subsection{Transceptor-mediated amino acid sensing in $C$. albicans and $C$. neoformans}

C. albicans expresses six orthologues of the S. cerevisiae GAP1 (97). Complementation experiments in the Scgap1 $1 \Delta$ strain showed that CaGap1, 2 and 6 can also function as transceptors as they are able to rapidly activate the PKA pathway upon addition of their substrates to nitrogen-starved cells (97) (Figure 2). Although the different $\mathrm{CaGap}$ permeases share high sequence similarity, substrate specificity differs substantially. It remains to be determined whether these proteins also function as transceptors in C. albicans.

In C. neoformans, there are at least seven ScGap1 homologs in the H99 genome database. However, the function of this gene group has not been analyzed in detail. It remains unknown whether there is any transceptor mediated amino acid sensing in C. neoformans.

\subsection{Transceptor-mediated amino acid sensing in filamentous fungi}

So far, no studies have shown the presence of transceptor-mediated amino acid sensing in filamentous fungi.

\subsection{Transceptor-mediated ammonium sensing in yeasts}

Ammonium is an important nitrogen source acquired from the breakdown of nitrogenous compounds or its specific uptake from the environment. A conserved family of transporters mediate ammonium import and some additionally act as transceptors (98-104). When levels of easily metabolizable nitrogen sources are low, or when cells are grown on a poor nitrogen source, fungi experience nitrogen limitation, resulting in reduced growth. During this nitrogen limitation, the ammonium transceptors regulate mating in $C$. neoformans and filamentous growth in $S$. cerevisiae, S. pombe, C. albicans, and U. maydis (98-104). The S. cerevisiae genome encodes three ammonium transporters (Mep1-3), however only Mep2 is a 
transceptor, acting as a regulator of pseudohyphal growth and PKA pathway activation when ammonium is reintroduced to nitrogen starved cells $(98,100,105)$. Expression of the MEP genes is regulated by NCR while the Mep2 protein is controlled by the TORC1 regulated Npr1 kinase $(106,107)$. Mep2 phosphorylation is not required for plasma membrane targeting and stability, but exerts a positive control on protein activity by initiating a conformational change in the cytoplasmic Cterminal domain of the protein $(107,108)$.

Ammonium transporters are homotrimers with each monomer forming a right-handed helical bundle that surrounds the pore through which ammonium translocation takes place. Substrate binding occurs at a discriminating extracellular site and two overlapping phenylalanine residues block the pore opening (108-112). Unlike bacterial homologs, the pores of Mep2 from both $S$. cerevisiae and $C$. albicans are closed on the cytoplasmic side of the protein (108). Molecular dynamics simulations support the deprotonation of the ammonium ion in bacterial transporters and the movement of ammonia gas and protons separately through the protein (113116). A similar mechanism of transport may occur in the fungal transceptors as they contain two histidine residues $\left.\left(\mathrm{His}_{1}-\mathrm{His}\right)_{2}\right)$ in the pore that may act as a proton relay system $(108,116)$. Non-sensing fungal ammonium transporters contain a glutamate and a histidine residue $\left(\mathrm{Glu}_{1}-\mathrm{His}_{2}\right)$ at the equivalent positions although the significance of this difference with regard sensing has not been established (117). Sensing is dependent on ammonium import through the transceptor and mutations have been identified that uncouple transport and signaling and that distinguish between PKA activation in starved cells and pseudohyphal induction $(105,118)$. The Mep2 $\mathrm{His}_{2}$ residue is particularly interesting as it is dispensable for transport, but essential for signaling and is involved in blocking the cytoplasmic side of the pore $(108,118)$. The potential importance of the $\mathrm{His}_{2}$ residue in signaling is supported by mutation of an adjacent residue that generates a hyperactive sensor (107).

Transceptor ammonium sensing does not reflect changes in internal nitrogen metabolism as the non-metabolizable ammonium analog methylamine induces the rapid activation of the PKA pathway and signaling mutants that import ammonium exist $(105,117)$. These findings suggest that it is the action of the protein as a transporter that is important for the sensing event. In one model the conformational changes that occur during the transport process could be channelled to a signaling partner. In support of this model, conformational changes occur in Mep2 in response to phosphorylation and the closed nature of the pore suggests additional conformational changes might occur during ammonium transport $(107,108)$. Furthermore, a physical interaction has been identified between the Ump2 receptor 
and the signaling protein Rho1 in U. maydis (119). In S. cerevisiae and C. albicans genetic interactions between Mep2 and the PKA signaling pathway have been identified $(100,101,118)$. It has also been proposed that signaling and non-signaling transporters may influence internal $\mathrm{pH}$ differently if one mediates electrogenic rather than electroneutral transport (117). In this model sensing would be dependent on a downstream $\mathrm{pH}$ sensor.

\subsection{Transceptor-mediated ammonium sensing in filamentous fungi}

The number of ammonium transceptors that have been studied suggests that this mode of signaling may be widespread in fungi. To support this, homologs from diverse fungi restore signaling in a $S$. cerevisiae strain lacking the Mep2 transceptor, including transporters from the filamentous rice pathogen Fusarium fujikuroi (MepA and $\mathrm{MepC}$ ), and the ectomycorrhizal fungus Hebeloma cylindrosporum (Amt1 and Amt2) $(120,121)$. Although these proteins are able to restore pseudohyphal growth in $S$. cerevisiae, the processes they regulate within their native organism remain unclear. In the broad range plant pathogen Colletotrichum gloeosporioides, transceptor-mediated ammonium sensing has been shown to be important for invasive growth and virulence (122).

\section{GPCRs}

G-protein coupled receptors (GPCRs) communicate changes in the extracellular environment to intracellular G-proteins, which initiate signaling events that coordinate the appropriate response. GPCR-ligand binding causes the dissociation of the heterotrimeric G-proteins into the Ga-subunit and a G $\beta y$-dimer, which activate downstream pathways (123). G-protein functions are transient, and regulated by repressors of G-protein signaling (RGS), which promote G-protein reassociation, and RGS ubiquitination/degradation, feedback mechanisms. Despite the general GPCR-signaling mechanism being highly conserved in eukaryotes, significant differences exist between yeasts and filamentous fungi. Five main categories of classical GPCRs exist, including pheromone, carbon, nitrogen, cAMP and microbial opsin receptors. Additional non-classical GPCRs include the orthologues of Pth11 (Magnoporthe oryzae), mPR (humans), a hormone receptor (rat) and AtRgs1 (Arabidopsis thaliana). However, relatively few GPCR-G-protein interactions in fungi are characterized and even fewer pathway-activating ligands have been identified.

\subsection{GPCR-mediated carbon sensing in S. cerevisiae}


Addition of various carbon sources, such as glucose, sucrose, mannose or fructose, to glucose-deprived cells triggers rapid activation of the cAMP-PKA pathway (124-126). PKA becomes activated due to an increase in cAMP, as a result of adenylate cyclase activation via a dual-glucose sensing system, extracellular detection through a GPCR as well as intracellular sensing dependent on sugar uptake and phosphorylation (127) (Figure 1). The activation of PKA results in the phosphorylation of a wide spectrum of target proteins associated with rapid cell growth (128).

Extracellular sugar sensing is mediated via the GPCR, Gpr1, the Go-protein Gpa2 (129) and its RGS protein, Rgs2 (130). Detailed analysis of the ScGpr1 receptor, using cysteine scanning mutagenesis and SCAM, identified specific amino acids in transmembrane domain six that bind glucose and sucrose as ligands thereby mediating the activation of the PKA pathway (125).

In addition to a role in activation of the PKA pathway, Gpr1 is required for pseudohyphal filamentous growth and also accounts for the glucose sensitivity of the mating pathway (131). The Sks1 kinase was recently identified as a novel component linking glucose sensing by Gpr1 and induction of genes involved in pseudohyphal differentiation (132). Gpa2 is an atypical $G \alpha$-protein, in that it is not part of a heterotrimeric complex, however some proteins have been identified as candidate G $\beta \gamma$-subunits. The Kelch-repeat proteins, Krh1/Gpb2 and Krh2/Gpb1 (133, 134) as well as Asc1, a protein binding to Gpa2 in a guanine nucleotide-dependent manner (135). The Krh proteins, activated by Gpa2, downregulate PKA by increasing the cAMP dependency (136). In this way, they are part of an adenylate cyclase bypass, allowing the direct activation of PKA by activated Gpa2. In addition, the Krh proteins provide a negative feedback loop by phosphorylating the PKA regulatory subunit, Bcy1, and hence promoting its association with the catalytic subunits (137, 138). Recently, Gpr1 was also shown to be required for the induction of the mating pathway (139).

\subsection{GPCR-mediated carbon sensing in C. albicans and C. neoformans}

In $C$. albicans, the situation regarding the number of GPCRs is similar to that of S. cerevisiae. There are two mating factor GPCRs and one orthologue of the ScGPR1, CaGpr1 (140-142). Deletion of CaGPR1 results in a strong morphogenetic defect on hyphae-inducing media, less damage in a reconstituted tissue infection model but only a slight effect on virulence in a systemic infection model system (141) (Figure 1). Contrary to the situation in S. cerevisiae, CaGpr1 is not required for the 
glucose-induced cAMP increase. Although the exact ligand still remains to be identified, it is possible that CaGpr1 is a glucose sensor that does not activate AC. Another possibility is that methionine is the ligand as it was shown that CaGpr1 is internalized in the presence of methionine (resembling the ligand-induced internalization known in other receptors) and there is no methionine-induced morphogenesis in the Cagpr1 mutant $(141,143)$. This latter phenotype is however only observed in the presence of low physiological concentrations of glucose, so it cannot be excluded that CaGpr1 is a low glucose sensor.

In C. neoformans, glucose is a preferred carbon source and a known signal to activate the homologous Gpa1-cAMP-PKA signal transduction pathway, which controls the production of major virulence factors (capsule and melanin) and is essential for fungal virulence (144). But it remains unknown how glucose activates Gpa1 and what is the GPCR for glucose sensing in C. neoformans. Multiple GPCR candidates have been identified based on the basic seven transmembrane domain structure, but none of them share extensive sequence identity with ScGpr1, except maybe Gpr4 (145). Both Gpr4 and ScGpr1 have a large third cytoplasmic loop and a long C-terminal tail. Despite the structural similarity, Gpr4 is not important for glucose sensing, but as mentioned below, is classified as a methionine receptor. Gpr5 also contains a large third intracellular loop and shares high sequence identity with Gpr4 and Gpr5 was found to play a role in regulating fungal cell size ("Titan" cell formation) during infection, likely by sensing certain unidentified host signals in the lung (146). Phylogenetic analysis based on proteins sequences shows that Gpr4 and Gpr5 are more closely related to $\mathrm{GprH}$ in A. nidulans and cAR1 in Dictyostelium discoideum, than with Gpr1 in S. cerevisiae (145). This is also consistent with the data indicating that Gpr4 and Gpr5 are not glucose receptors. How glucose is sensed remains to be understood. It is possible that there is functional redundancy among GPCRs in glucose sensing that complicates the identification of glucose receptors.

\subsection{GPCR-mediated carbon sensing in filamentous fungi}

In contrast to yeasts, filamentous fungi can utilize a diverse array of nutrient sources including hexose, pentose and complex saccharides. This nutritional plasticity may therefore be reflected in the presence of additional Ga-proteins and the dramatic expansion of putative GPCRs (123).

In N. crassa, the putative carbon sensor, Gpr4, interacts with the G-protein, Gna1, influencing cAMP production and asexual development in a carbon sourcedependent manner (147) (Figure 1). The absence of Gpr4 resulted in reduced growth on glycerol, mannitol, and arabinose, a phenotype that was partial recovered via the 
addition of exogenous cAMP. In $A$. nidulans, a putative carbon receptor, GprD, influences hyphal growth, germination and primary metabolism (148). Interestingly, GprD and the pheromone receptor, GprB, possess opposing functions in the regulation of sexual development (149). Similarly, the $A$. nidulans cAMP receptor-like GPCR, GprH, was induced during carbon starvation and was shown to be involved in glucose and amino acid sensing, while influencing primary metabolism via the cAMPPKA pathway and acting as a repressor of sexual development (150). In Aspergillus fumigatus, two putative carbon receptors, GprC and GprD, were fundamental to germination, hyphal extension and branching (151). Furthermore, the absence of GprC or GprD increased sensitivity toward environmental stress caused by reactive oxygen intermediates and displayed attenuated virulence in a murine infection model. Transcriptome analyses indicated that both GprC and GprD promoted primary metabolism, while inhibiting secondary metabolism (151). A wider evaluation of the function of all 15 classical GPCRs in Aspergillus flavus revealed multiple GPCRs potentially involved in carbon sensing, as the individual absence of GprA, GprC, GprJ, GprK or GprR impaired growth on several carbon sources (152). Therefore, in contrast to yeasts, filamentous fungi appear to possess multiple GPCRs involved in sensing a broad range of carbon sources, reflecting the complexity of the environments that they inhabit, their metabolic plasticity and their capacity to utilize a diverse array carbon sources.

\subsection{GPCR-mediated amino acid sensing by GPCRs in C. albicans and C.} neoformans

Apart from the above mentioned amino acid receptors, some GPCRs have also been reported to sense amino acids in fungi. In C. albicans, Gpr1, the homolog of the glucose sensor ScGpr1, has been suggested as a possible receptor for methionine. Methionine is important for the yeast-to-hyphal transition on sold agar

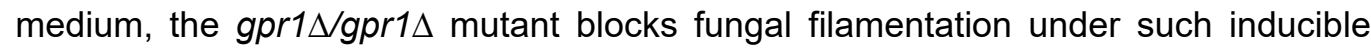
condition. Gpr1 senses methionine to activate the cAMP-PKA pathway, which in turn controls the filamentation in the presence of glucose as a carbon source $(141,143)$. It is however not yet excluded that CaGpr1 may actually sense low (or physiological) glucose levels.

In C. neoformans, the GPCR protein Gpr4 also plays a role in sensing amino acids to activate CAMP-PKA signaling (145) (Figure 2). Mutagenesis demonstrated that Gpr4 is required for capsule production and fungal mating, two cellular functions controlled by the Gpa1-cAMP-PKA pathway. The direct interaction between Gpr4 and Gpa1 G-protein has also been confirmed in a membrane based yeast two-hybrid 
system. Methionine can trigger Gpr4 internalization and induces a transient cAMP production in a Gpr4-dependent manner. Also a low level of methionine can induce mating hyphal elongation in C. neoformans, which is blocked by the deletion of GPR4. This data indicates that Gpr4 may function as a receptor for amino acids, such as methionine (145). However, the direct binding of amino acids to CaGpr1 or CnGpr4 has not been observed. Whether these GPCRs sense extracellular, or intracellular amino acid, or both, also remains to be determined.

\subsection{GPCR-mediated amino acid sensing in filamentous fungi}

GPCRs, in filamentous fungi, have been reported to sense amino acids. The cAMP receptor-like GPCR, in A. nidulans, GprH, shows distant homology to the cAMP receptors of $D$. discoideum and $A$. thaliana, as well as to Gpr4 in $C$. neoformans (123). Nonetheless, GprH was revealed to be a putative receptor for both glucose and the amino acid tryptophan, forming part of the cAMP-PKA pathway, and influencing primary metabolism, while repressing sexual development, during carbon starvation $(150,153)$. GprH also shows homology to the putative $N$. crassa carbon receptor, Gpr4. The absence of this receptor caused defects in perithecial development and ascospore discharge. However, the binding of this putative ligand was not defined (154). Therefore, the function of cAMP receptor-like GPCRs in regulating sexual development appears to be conserved in fungi, while receptors of this class may dually detect both sugars and amino acids. Additionally, in A. flavus, the absence of the putative carbon receptors, GprC and GprD, which showed homology to the S. pombe glucose GPCR Git3, inhibited growth on the amino acid, proline (152). Hence, filamentous fungal GPCRs of several classes may potentially influence various amino acid and carbon nutrient sensing pathways.

\subsection{GPCR-mediated ammonium sensing in filamentous fungi}

In contrast to yeasts, very little is known of ammonium sensing in filamentous fungi. The $A$. flavus genome encodes four receptors that show homology to the $S$. pombe Stm1 sensor, which regulates cell cycle progression in response to nitrogen starvation (155). The absence of one of these receptors, GprR, impaired growth on ammonium chloride and the amino acid, proline (152). This again, suggests that in

filamentous fungi a single GPCR may play a role in multiple nutrient sensing pathways.

\subsection{GPCR-mediated host sensing in filamentous fungal pathogens}


In filamentous fungi, GPCR-regulated signaling pathways include the cAMPdependent PKA and the MAPK cascades, which regulate growth, metabolism, mating, morphogenesis, cell division, cell-to-cell fusion, chemotaxis, toxicogenesis and virulence (156). It is increasingly becoming apparent that GPCRs can bind multiple ligands and in turn possess additional functions. The vascular root phytopathogen, Fusarium oxysporum, demonstrates chemotaxis towards specific nutrients (glucose, glutamate and aspartate, but not glutamine, methionine, ammonium or galactose) and host tomato plant root exudates, in particular a class II peroxidase that is associated with root hairs which represent a potential site for invasion (157). Chemotropic sensitivity to the a-pheromone is far greater than nutrients, suggesting that the two responses are mediated by distinct mechanisms. Accordingly, mutants in the Fmk1 filamentous growth pathway are impaired in their response to glutamate or glucose, but not the a-pheromone, while mutants in the Mpk1 cell wall integrity pathway are impaired in their chemotaxis towards the $\alpha$ pheromone, but not glutamate or glucose. The absence of Ste2/Pre2 pheromone receptor in $F$. oxysporum abolishes chemotropism towards the a-pheromone and tomato root exudates, while having a minor impact on virulence, demonstrating how Ste2 GPCR-mediated signaling, which was previously thought to be exclusively involved in pheromone sensing, is also involved in detecting host cues and promoting virulence (157).

In the foliar rice pathogen, $M$. oryzae, the Gaßy-subunits and the nonclassical GPCR, Pth11, sense surface hydrophobicity and plant cutin monomers, regulating appressoria formation and virulence in a cAMP-dependent manner (158). Evaluation of the spatio-temporal dynamics of G-protein signaling during pathogenic development showed key components, including Pth11, MagA, Rgs1 and the adenylate cyclase, to be sequestered to the tubulo-vesicular network, where late endosomes controlled the geometry and activation/de-activation of the cAMP signal during pathogenesis (159). Subsequently, numerous Pth11-related receptors have been identified in $M$. oryzae and other filamentous fungi from the ascomycete subphyla, Pezizomycotina, including $A$. nidulans, $N$. crassa and Fusarium graminearum, while none were found in other ascomycete subphyla, such as the yeasts, or the basidiomycetes $(123,160)$. Hence, this non-classical receptor class represents the most highly expand receptor class in filamentous ascomycete fungi. In turn, a recent analysis of the classical and non-classical GPCRs in $N$. crassa, identified numerous Pth11 homologs and revealed their involvement in nutrient sensing. The absence of three Pth11 homologs in N. crassa, Gpr32, Gpr36 and Gpr39, enhanced growth on cellulose as an alternative to sucrose (161). Therefore, 
in filamentous fungi Pth11-like receptors appear to participate in sensing plantrelated compounds, applicable to a saprophytic and a pathogenic interaction with plants.

\section{Extracellular mucin receptors in fungi}

\subsection{Extracellular mucin receptors in S. cerevisiae}

Exposure of $S$. cerevisiae to nitrogen deprivation activates pseudohyphal and invasive growth, in addition to biofilm formation, via activating MAPK signaling to enable nutrient foraging and survival (162-165). Extracellular signaling mucins act as sensors of nutrient availability and physical contact, regulating the connection between cells, or to a surface (Figure 3 ). Fungal and humans signaling mucins utilize a highly conserved induction mechanism that results in GTPase activation (166). Nutrient deprivation promotes the proteolytic cleavage of an extracellular inhibitory mucin domain, activating intracellular Msb2-mediated MAPK signaling (162, 167). The highly glycosylated extracellular mucin domain of Msb2 is cleaved off by the Yps1 yapsin protease, releasing the inhibitory domain into the surrounding environment to create a mucous glycoprotein layers around cells, analogs of MUC1/2 mucins in humans $(166,167)$. Upon starvation, the Mig1 carbon catabolite repressor that inhibits alternative carbon usage is phosphorylated by Snf1, promoting its dissociation from the DNA, its migration from the nucleus, and its interaction with multiple members of the pseudohyphal MAPK cascade, including Msb2, which influences filamentation (164). The Snf1-Mig1 mechanisms for preferential carbon usage is therefore interlinked to the pseudohyphal MAPK pathway, coordinating cellular metabolism and proliferation in response to nutrient availability (164). Cell adhesion is also controlled by the Ras, CAMP-PKA and MAPK pathways in response to nutrient deprivation and other exogenous stresses (168), while adhesion impacts upon pseudohyphal growth and biofilm formation (169, 170). The Msb2pseudohyphal MAPK pathway regulates the secretion of the Flo11 flocculin, which is essential for adhesion and pseudohyphal growth (169). Therefore, in response to nutrient availability, Msb2-MAPK signaling induces filamentous growth, cell adhesion and biofilm formation to promote nutrient foraging and survival.

\subsection{Extracellular mucin receptors in C. albicans}

The regulation of MAPK cascades by Msb2, controlling filamentous growth, is conserved in C. albicans, where shedding of Msb2 and the induction of MAPK signaling, promotes filamentous growth and influences biofilm formation $(171,172)$ 
(Figure 3). In C. albicans, Msb2, in cooperation with Sho1, controls the activation of the Cek1 MAPK under cell wall or osmotic stress. The absence of Msb2 had a stronger impact on invasive growth on certain solid media such as mannitol or sucrose, and under hypoxia, than the loss of Sho1 (171). Similar to S. cerevisiae, in C. albicans the Sap8 aspartic protease cleaves the extracellular Msb2 mucin domain, activating Cek1 signaling and biofilm formation (172). The absence of Msb2 or Sap8 resulted in the increased exposure of $\beta$-glucans and the loss of Msb2 attenuated virulence in a murine model of oral candidiasis. Additionally, Msb2 shedding also protects against antimicrobial peptides $(173,174)$. Therefore, in addition to regulating invasive growth and biofilm formation, the Msb2 pathway in C. albicans performs an essential function in reducing the exposure of pathogen-associated molecular patterns that trigger host defenses and increasing the tolerance of exogenous stresses such as antimicrobial peptides, by creating a mucous layer. However, the nutritional and/ or environmental signals that promote Msb2 signaling remain to be determined in C. albicans.

\subsection{Extracellular mucin receptors in filamentous fungi}

Surface recognition is one of the most critical processes for both saprophytic and pathogenic filamentous fungi. In the heterogeneous semi-solid environment, naturally occupied by fungi, the majority of nutrients are locked away as insoluble biomass. Hence, fungi have to actively search for, and attach to a desirable source of nutrients. Subsequently, during this foraging period starvation and contact sensing are hypothesized to participate in the localization and deconstruction of complex nutrient sources. In the lignocellulolytic fungus, $A$. nidulans, the extracellular mucin, $\mathrm{MsbA}$, influences growth under nutrient-poor conditions including that on lignocellulose. Through the modulation of both the cell wall integrity and filamentous growth MAPK cascades, MsbA regulates adhesion to solid surfaces, biofilm formation and lignocellulolytic enzyme secretion (175). In particular, the CbhA cellobiohydrolase required to release cellobiose from lignocellulose and the induction of the lignocellulolytic machinery. Therefore, starvation and/ or surface sensing represent a new dimension to the already multifaceted regulation of nutrient sensing, CAZyme production and lignocellulose deconstruction (Figure 3).

In the case of filamentous phytopathogens, the recognition of host surfaces, and their subsequent penetration, is paramount for the establishment of infection. The filamentous growth MAPK pathway is central to the establishment of invasive growth and virulence (156). In the vascular root pathogen, F. oxysporum, Msb2 induces the phosphorylation of Fmk1, which promotes cellophane and tomato root 
penetration, contributing to fungal virulence (176). Similarly, the F. oxysporum Sho1 receptor was also required for Fmk1 phosphorylation, extracellular pectinolytic activity, cellophane penetration, plant tissue colonization and virulence (177). The dimorphic pathogen of maize, U. maydis, switches from budding to hyphal growth and the development of appressoria in response to the detection of the hydrophobic, waxy, plant surfaces. In U. maydis the interacting Msb2 and Sho1 receptors function upstream of the Kpp2-Kpp6 MAPK cascade, and are key to the development of appressoria in response to hydrophobic surfaces, in addition to priming the fungus for biotrophic development $(178,179)$. These examples of the overlapping functionality of Msb2 and Sho1 suggest a genetic interaction between the two receptors, which was noted in yeasts, is conserved in filamentous fungi.

In the foliar rice pathogen, $M$. oryzae, multiple surface sensors detect rice leaf surfaces, appressoria formation and host invasion, including the aforementioned non-classical GPCR, Pth11, plus the Msb2, Sho1 and Cbp1 receptors (158, 180, 181). Similar to Pth11, the absence of Msb2 significantly reduced appressoria formation and virulence, while the absence of Sho1 only resulted in a slight reduction in virulence. The dual absence of Msb2 Sho1 resulted in an inability to respond to cutin monomers and an inability to form appressoria on artificial hydrophobic surfaces. Appressoria formation on hydrophobic surfaces, leaf waxes or primary alcohols was more severely inhibited in the dual absence of Msb2 and Sho1 than in the absence of just Msb2. This suggests that the two interacting receptors possess overlapping functions, yet Msb2 is critical for sensing surface hydrophobicity and cutin monomers, while Sho1 appears to be important in recognizing rice leaf waxes. Therefore, in addition to surface hydrophobicity and cutin monomers, primary alcohols in leaf epicuticular waxes are utilized as signals for fungal infection (180). $M$. oryzae possesses another putative mucin, CBP1 (named for chitin-binding protein 1 ), and the absence of $\mathrm{Cbp} 1$ also resulted in an inability to produce appressoria on hydrophobic surfaces, while retaining the ability to form appressoria on leaves (182), potentially due to the action of Msb2. In accordance, the absence of both the extracellular mucins, Msb2 and Cbp1, blocked Pmk1 MAPK phosphorylation, impeded appressoria formation and abolished virulence, suggesting that the Msb2 and Cbp1 mucins have overlapping functions in the recognition of environmental cues required for the penetration of host tissues (181). Therefore, in filamentous fungi the extracellular mucin and Sho1 receptors appear to have evolved the ability to detect the hydrophobic, waxy, alcohol composition of plant surfaces and in doing so perform a fundamental role in plant pathogenesis. 


\section{Membrane-localized transceptors or receptors link the availability of nutrients to downstream signal transduction pathways}

In this chapter we described the nature of the receptors by which different nutrients are sensed by fungi. We distinguished between non-transporting sugar or nitrogen receptors, sugar or nitrogen transceptors, sugar, nitrogen and host sensing GPCRs as well as Mucin receptors. In our chapter, we briefly mentioned the signal transduction pathways that are activated upon the sensing of nutrients by these receptors. Figure 4 gives an overview of what is currently known about the downstream signaling pathways, which we also briefly touched upon. The two most important downstream pathways are the MAPK and CAMP-PKA pathways, which are not only involved in nutrient sensing but are also involved in mating (see Chapter $X X X)$. Non-transporting receptors and some transceptors regulate the expression of nutrient transporters upon sensing that specific nutrient. A detailed description of these pathways is described in other chapters in this Fungal Kingdom book.

\section{References}

1. Özcan S, Johnston M. 1999. Function and regulation of yeast hexose transporters. Microbiol Mol Biol Rev 63:554-569.

2. Özcan S, Dover J, Rosenwald AG, Wolfl S, Johnston M. 1996. Two glucose transporters in Saccharomyces cerevisiae are glucose sensors that generate a signal for induction of gene expression. Proc Natl Acad Sci U S A 93:1242812432.

3. Karhumaa K, Wu B, Kielland-Brandt M. 2010. Conditions with high intracellular glucose inhibit sensing through glucose sensor Snf3 in Saccharomyces cerevisiae. J Cell Biochem 110:920-925.

4. Cairey-Remonnay A, Deffaud J, Wésolowski-Louvel M, Lemaire M, Soulard A. 2015. Glycolysis controls plasma-membrane glucose sensors to promote glucose signaling in yeasts. Mol Cell Biol 35:747-757.

5. Özcan S, Dover J, Johnston M. 1998. Glucose sensing and signaling by two glucose receptors in the yeast Saccharomyces cerevisiae. EMBO J 17:25662573. 
6. Polish JA, Kim JH, Johnston M. 2005. How the Rgt1 transcription factor of Saccharomyces cerevisiae is regulated by glucose. Genetics 169:583-594.

7. Kim JH, Polish J, Johnston M. 2003. Specificity and regulation of DNA binding by the yeast glucose transporter gene repressor Rgt1. Mol Cell Biol 23:52085216.

8. Palomino A, Herrero P, Moreno F. 2006. Tpk3 and Snf1 protein kinases regulate Rgt1 association with Saccharomyces cerevisiae HXK2 promoter. Nucleic Acids Res 34:1427-1438.

9. Kaniak A, Xue Z, Macool D, Kim JH, Johnston M. 2004. Regulatory network connecting two glucose signal transduction pahtways in Saccharomyces cerevisiae. Eukaryot Cell 3:221-231.

10. Moriya H, Johnston M. 2004. Glucose sensing and signaling in Saccharomyces cerevisiae through the Rgt2 glucose sensor and casein kinase I. Proc Natl Acad Sci U S A 101:1572-1577.

11. Spielewoy N, Flick K, Kalashnikova TI, Walker JR, Wittenberg C. 2004. Regulation and recognition of SCFGrr1 targets in the glucose and amino acid signaling pathways. Mol Cell Biol 24:8994-9005.

12. Kim JH, Johnston M. 2006. Two glucose-sensing pathways converge on Rgt1 to regulate expression of glucose transporter genes in Saccharomyces cerevisiae. J Biol Chem 281:26144-26149.

13. Roy A, Jouandot Dn, Cho KH, Kim JH. 2014. Understanding the mechanism of glucose-induced relief of Rgt1-mediated repression in yeast. FEBS Open Bio 4:105-111.

14. Betina S, Goffrini P, Ferrero I, Wésolowski-Louvel M. 2001. RAG4 gene encodes a glucose sensor in Kluyveromyces lactis. Genetics 158:541-548.

15. Chen XJ, Wésolowski-Louvel M, Fukuhara H. 1992. Glucose transport in the yeast Kluyveromyces lactis. II. Transcriptional regulation of the glucose transporter gene RAG1. Mol Gen Genet 233:97-105.

16. Blaisonneau J, Fukuhara H, Wésolowski-Louvel M. 1997. The Kluyveromyces lactis equivalent of casein kinase I is required for the transcription ofthe gene encoding the low-affinity glucose permease. Mol Gen Genet 253:469-477.

17. Hnatova M, Wésolowski-Louvel M, Dieppois G, Deffaud J, Lemaire M. 2008. Characterization of KIGRR1 and SMS1 genes, two new elements of the glucose signaling pathway of Kluyveromyces lactis. Eukaryot Cell 7:1299-1308. 
18. Cotton P, Soulard A, Wésolowski-Louvel M, Lemaire M. 2012. The SWI/SNF $\mathrm{KISnf} 2$ subunit controls the glucose signaling pathway to coordinate glycolysis and glucose transport in Kluyveromyces lactis. Eukaryot Cell 11:1382-1390.

19. Stasyk OG, Maidan MM, Stasyk OV, Van Dijck P, Thevelein JM, Sibirny AA. 2008. Identification of hexose transporter-like sensor HXS1 and functional hexose transporter HXT1 in the methylotrophic yeast Hansenula polymorpha. Eukaryot Cell 7:735-746.

20. Fan J, Chaturvedi V, Shen SH. 2002. Identification and phylogenetic analysis of a glucose transporter gene family from the human pathogenic yeast Candida albicans. J Mol Evol 55:336-346.

21. Brown V, Sexton JA, Johnston M. 2006. A glucose sensor in Candida albicans. Eukaryot Cell 5:1726-1737.

22. Sabina J, Brown V. 2009. Glucose sensing network in Candida albicans: a sweet spot for fungal morphogenesis. Eukaryot Cell 8:1314-1320.

23. Bahn YS, Xue C, Idnurm A, Rutherford JC, Heitman J, Cardenas ME. 2007. Sensing the environment: lessons from fungi. Nat Rev Microbiol 5:57-69.

24. Liu TB, Wang Y, Baker GM, Fahmy H, Jiang L, Xue C. 2013. The glucose sensor-like protein Hxs1 is a high-affinity glucose transporter and required for virulence in Cryptococcus neoformans. PLoS One 8:e64239.

25. Xue C. 2012. Cryptococcus and beyond--inositol utilization and its implications for the emergence of fungal virulence. PLoS Pathog 8:e1002869.

26. Healy ME, Dillavou CL, Taylor GE. 1977. Diagnostic medium containing inositol, urea, and caffeic acid for selective growth of Cryptococcus neoformans. J Clin Microbiol 6:387-391.

27. Xue C, Tada Y, Dong X, Heitman J. 2007. The human fungal pathogen Cryptococcus can complete its sexual cycle during a pathogenic association with plants. Cell Host Microbe 1:263-273.

28. Fisher SK, Novak JE, Agranoff BW. 2002. Inositol and higher inositol phosphates in neural tissues: homeostasis, metabolism and functional significance. J Neurochem 82:736-754.

29. Xue C, Liu T, Chen L, Li W, Liu I, Kronstad JW, Seyfang A, Heitman J. 2010. Role of an expanded inositol transporter repertoire in Cryptococcus neoformans sexual reproduction and virulence. mBio 1:e00084-00010.

30. Liu TB, Subbian S, Pan W, Eugenin E, Xie J, Xue C. 2014. Cryptococcus inositol utilization modulates the host protective immune response during brain infection. Cell Commun Signal 12:51. 
31. Liu TB, Kim JC, Wang Y, Toffaletti DL, Eugenin E, Perfect JR, Kim KJ, Xue C. 2013. Brain inositol is a novel stimulator for promoting Cryptococcus penetration of the blood-brain barrier. PLoS Pathog 9:e1003247.

32. Wang Y, Liu TB, Delmas G, Park S, Perlin D, Xue C. 2011. Two major inositol transporters and their role in cryptococcal virulence. Eukaryot Cell 10:618-628.

33. Brown NA, Ries LNA, Goldman GH. 2014. How nutritional status signalling coordinates metabolism and lignocellulolytic enzyme secretion. Fungal Genet Biol 72:48-63.

34. Zhang WX, Kou YB, Xu JT, Cao YL, Zhao GL, Shao J, Wang H, Wang ZX, Bao XM, Chen GJ, Liu WF. 2013. Two Major Facilitator Superfamily Sugar Transporters from Trichoderma reesei and their roles in induction of cellulase biosynthesis. J Biol Chem 288:32861-32872.

35. Znameroski EA, Coradetti ST, Roche CM, Tsai JC, lavarone AT, Cate JHD, Glass NL. 2012. Induction of lignocellulose-degrading enzymes in Neurospora crassa by cellodextrins. Proc Natl Acad Sci USA 109:6012-6017.

36. Cai PL, Wang B, Ji JX, Jiang YS, Wan L, Tian CG, Ma YH. 2015. The Putative Cellodextrin Transporter-like Protein CLP1 Is Involved in Cellulase Induction in Neurospora crassa. J Biol Chem 290:788-796.

37. Forsberg H, LjungdahI PO. 2001. Sensors of extracellular nutrients in Saccharomyces cerevisiae. Curr Genet 40:91-109.

38. Kodama Y, Omura F, Takahashi K, Shirahige K, Ashikari T. 2002. Genomewide expression analysis of genes affected by amino acid sensor Ssy1p in Saccharomyces cerevisiae. Curr genet 41:63-72.

39. Klasson H, Fink GR, LjungdahI PO. 1999. Ssy1p and Ptr3p are plasma membrane components of a yeast system that senses extracellular amino acids. Mol Cell Biol 19:5405-5416.

40. Wu B, Ottow K, Poulsen P, Gaber RF, Albers E, Kielland-Brandt MC. 2006. Competitive intra- and extracellular nutrient sensing by the transporter homologue Ssy1p. J Cell Biol 173:327-331.

41. Gaber RF, Ottow K, Andersen HA, Kielland-Brandt M. 2003. Constitutive and hyperresponsive signaling by mutant forms of Saccharomyces cerevisiae amino acid sensor Ssy1. Eukaryot Cell 2:922-929.

42. Poulsen P, Gaber RF, Kielland-Brandt MC. 2008. Hyper- and hyporesponsive mutant forms of the Saccharomyces cerevisiae Ssy1 amino acid sensor. Mol Membr Biol 25:164-176.

43. Souciet J, Aigle M, Artiguenave F, Blandin G, Bolotin-Fukuhara M, Bon E, Brottier P, Casaregola S, de Montigny J, Dujon B, Durrens P, Gaillardin C, 
Lépingle A, Llorente B, Malpertuy A, Neuvéglise C, Ozier-Kalogéropoulos O, Potier S, Saurin W, Tekaia F, Toffano-Nioche C, Wésolowski-Louvel M, Winckler P, Weissenbach J. 2000. Genomic exploration of the hemiascomycetous yeasts. 1. A set of yeast species for molecular evolution studies. FEBS Lett 487:12540-12542.

44. Liu Z, Thornton J, Spirek M, Butow RA. 2008. Activation of the SPS amino acid-sensing pathway in Saccharomyces cerevisiae correlates with the phosphorylation state of a sensor component, Ptr3. Mol Cell Biol 28:551-563.

45. Omnus DJ, LjungdahI PO. 2013. Rts1-protein phosphatase 2A antagonizes Ptr3-mediated activation of the signaling protease Ssy5 by casein kinase I. Mol Biol Cell 24:1480-1492.

46. Maggio R, Innamorati G, Parenti M. 2007. G protein-coupled receptor oligomerization provides the framework for signal discrimination. J Neurochem 103:1741-1752.

47. Lemmon MA, Schlessinger J. 2010. Cell signaling by receptor tyrosine kinases. Cell 141:1117-1134.

48. Andréasson C, Heessen S, LjungdahI PO. 2006. Regulation of transcription factor latency by receptor-activated proteolysis. Genes Dev 20:1563-1568.

49. Pfirrmann T, Heessen S, Omnus DJ, Andréasson C, LjungdahI PO. 2010. The prodomain of Ssy5 protease controls receptor-activated proteolysis of transcription factor Stp1. Mol Cell Biol 30:3299-3309.

50. Abdel-Sater F, Jean C, Merhi A, Vissers S, André B. 2011. Amino acid signaling in yeast: activation of Ssy5 protease is associated with its phosphorylation-induced ubiquitylation. J Biol Chem 286:12006-12015.

51. Omnus DJ, Pfirrmann T, Andréasson C, LjungdahI PO. 2011. A phosphodegron controls nutrient-induced proteasomal activation of the signaling protease Ssy5. Mol Biol Cell 22:2754-2765.

52. Johnston M, Kim JH. 2005. Glucose as a hormone: receptor-mediated glucose sensing in the yeast Saccharomyces cerevisiae. Biochem Soc Trans 33:247252.

53. Andréasson C, LjungdahI PO. 2002. Receptor-mediated endoproteolytic activation of two transcription factors in yeast. Genes Dev 16:3158-3172.

54. Wielemans K, Jean C, Vissers S, André B. 2010. Amino acid signaling in yeast: post-genome duplication divergence of the Stp1 and Stp2 transcription factors. J Biol Chem 285:855-865. 
55. Tumusiime S, Zhang C, Overstreet MS, Liu Z. 2011. Differential regulation of transcription factors Stp1 and Stp2 in the Ssy1-Ptr3-Ssy5 amino acid sensing pathway. J Biol Chem 286:4620-4631.

56. Eckert-Boulet N, Larsson K, Wu B, Poulsen P, Regenberg B, Nielsen J, Kielland-Brandt MC. 2006. Deletion of RTS1, encoding a regulatory subunit of protein phosphatase $2 \mathrm{~A}$, results in constitutive amino acid signaling via increased Stp1p processing. Eukaryot Cell 5:174-179.

57. Andréasson C, LjungdahI PO. 2004. The N-terminal regulatory domain of Stp $1 \mathrm{p}$ is modular and, fused to an artificial transcription factor, confers full Ssy1p-Ptr3p-Ssy5p sensor control. Mol Cell Biol 24:7503-7513.

58. Boban M, LjungdahI PO. 2007. Dal81 enhances Stp1- and Stp2-dependent transcription necessitating negative modulation by inner nuclear membrane protein Asi1 in Saccharomyces cerevisiae. Genetics 176:2087-2097.

59. Omnus DJ, LjungdahI PO. 2014. Latency of transcription factor Stp1 depends on a modular regulatory motif that functions as cytoplasmic retention determinant and nuclear degron. Mol Biol Cell 25:3823-3833.

60. Shin CS, Kim SY, Huh WK. 2009. TORC1 controls degradation of the transcription factor Stp1, a key effector of the SPS amino-acid-sensing pathway in Saccharomyces cerevisiae. J cell Sci 122:2089-2099.

61. Brega E, Zufferey R, Mamoun BC. 2004. Candida albicans Csy1 is a nutrient sensor important for activation of amino acid uptake and hyphal morphogenesis. Eukaryotic Cell 3:135-143.

62. Martinez P, LjungdahI PO. 2004. An ER packaging chaperone determines the amino acid uptake capacity and virulence of Candida albicans. Mol Microbiol 51:371-384.

63. Martinez $\mathbf{P}$, Ljungdahl PO. 2005. Divergence of Stp1 and Stp2 transcription factors in Candida albicans places virulence factors required for proper nutrient acquisition under amino acid control. Mol Cell Biol 25:9435-9446.

64. Dabas N, Morschhäuser J. 2008. A transcription factor regulatory cascade controls secreted aspartic protease expression in Candida albicans. Mol Microbiol 69:586-602.

65. Davis MM, Alvarez FJ, Ryman K, Holm AAA, Ljungdahl PO, Engström Y. 2011. Wild-type Drosophila melanogaster as a model host to analyze nitrogen source dependent virulence of Candida albicans. PLoS One 6:e27434.

66. Fernandes JD, Martho K, Tofik V, Vallim MA, Pascon RC. 2015. The role of amino acid permeases and tryptophan biosynthesis in Cryptococcus neoformans survival. PLoS One 10:e0132369. 
67. Reifenberger E, Boles E, Ciriacy M. 1997. Kinetic characterization of individual hexose transporters of Saccharomyces cerevisiae and their relation to the triggering mechanisms of glucose repression. Eur J Biochem 245:324-333.

68. Bandyophadhyay G, Sajan MP, Kanoh Y, Standaert ML, Burke TRJ, Quon MJ, Reed BC, Dikic I, Noel LE, Newgard CB, Faresse R. 2000. Glusose activates mitogen-activated protein kinase (extracellular signal-regulated kinase) through proline-rich tyrosine kinase-2 and the Glut1 glucose transporter. J Biol Chem 275:40817-40826.

69. Guilleman G, Loizeau M, Pincon-Taymond M, Girard J, Leturque A. 2000. The large intracytoplasmic loop of the glucose transporter GLUT2 is involved in glucose signaling in hepatic cells. J cell Sci 113:841-847.

70. Stolarczyk E, Guissard C, Michau A, Even PC, Grosfeld A, Serradas P, Lorsignol A, Pénicaud L, Brot-Laroche E, Leturque A, Le Gall M. 2010. Detection of extracellular glucose by GLUT2 contributes to hypothalamic control of food intake. Am J Physiol Endocrinol Metab 298:E1078-1087.

71. Shewan AM, McCann RK, Lamb CA, Stirrat L, Kioumourtzoglou D, Adamson IS, Verma S, James DE, Bryant NJ. 2013. Endosomal sorting of GLUT4 and Gap1 is conserved between yeast and insulin-sensitive cells. J cell Sci 126:1576-1582.

72. Luo L, Tong X, Farley PC. 2007. The Candida albicans gene HGT12 (orf19.7094) encodes a hexose transporter. FEMS Immunol Med Microbiol 51:14-17

73. Luongo M, Porta A, Maresca B. 2005. Homology, disruption and phenotypic analysis of CaGS Candida albicans gene induced during macrophage infection. FEMS Immunol Med Microbiol 45:471-478.

74. Stasyk OV, Stasyk OG, Komduur J, Veenhuis M, Cregg JM, Sibirny AA. 2004. A hexose transporter homologue controls glucose repression in the methylotrophic yeast Hansenula polymorpha. J Biol Chem 279:8116-8125.

75. Madi L, McBride SA, Bailey LA, Ebbole DJ. 1997. rco-3, a gene involved in glucose transport and conidiation in Neurospora crassa. Genetics 146:499-508.

76. Lingner U, Münich S, Deising HB, Sauer N. 2011. Hexose transporters of a hemibiotrophic plant pathogen: functional variations and regulatory differences at different stages of infection. J Biol Chem 286:20913-20922.

77. Schuler D, WahI R, Wippel K, Vranes M, Munsterkotter M, Sauer N, Kamper J. 2015. Hxt1, a monosaccharide transporter and sensor required for virulence of the maize pathogen Ustilago maydis. New Phytol 206:1086-1100. 
78. Znameroski EA, Li X, Tsai JC, Galazka JM, Glass NL, Cate JHD. 2014. Evidence for Transceptor Function of Cellodextrin Transporters in Neurospora crassa. J Biol Chem 289:2610-2619.

79. Galazka JM, Tian C, Beeson WT, Martinez B, Glass NL, Cate JHD. 2010. Cellodextrin Transport in Yeast for Improved Biofuel Production. Science 330:84-86

80. Ha S-J, Galazka JM, Kim SR, Choi J-H, Yang X, Seo J-H, Glass NL, Cate JHD, Jin Y-S. 2011. Engineered Saccharomyces cerevisiae capable of simultaneous cellobiose and xylose fermentation. Proc Natl Acad Sci USA 108:504-509.

81. Regenberg B, Düring-Olsen L, Kielland-Brandt M, Holmberg S. 1999. Substrate specificity and gene expression of the amino-acid permeases in Saccharomyces cerevisiae. Curr genet 36:317-328.

82. Jauniaux JC, Grenson M. 1990. GAP1, the general amino acid permease gene of Saccharomyces cerevisiae. Nucleotide sequence, protein similarity with the other bakers yeast amino acid permeases, and nitrogen catabolite repression. Eur J Biochem 190:39-44.

83. Uemura T, Kashiwagi K, Igarashi K. 2005. Uptake of putrescine and spermidine by Gap1p on the plasma membrane in Saccharomyces cerevisiae. Biochem Biophys Res Commun 328:1028-1033.

84. Hofman-Bang J. 1999. Nitrogen catabolite repression in Saccharomyces cerevisiae. Mol Biotechnol 12:35-73.

85. Magasanik B, Kaiser CA. 2002. Nitrogen regulation in Saccharomyces cerevisiae. Gene 290:1-18.

86. De Craene JO, Soetens O, André B. 2001. The Npr1 kinase controls biosynthetic and endocytic sorting of the yeast Gap1 permease. J Biol Chem 276:43939-43948

87. Soetens O, De Craene JO, André B. 2001. Ubiquitin is required for sorting to the vacuole of the yeast general amino acid permease, Gap1. J Biol Chem 276:43949-43957.

88. O'Donnell AF, Apffel A, Gardner RG, Cyert MS. 2010. Alpha-arrestins Aly1 and Aly2 regulate intracellular trafficking in response to nutrient signaling. Mol Biol Cell 21:3552-3566.

89. Merhi A, Gérard N, Lauwers E, Prévost M, André B. 2011. Systematic mutational analysis of the intracellular regions of yeast Gap1 permease. PLoS One 6:e18457. 
90. Van Zeebroeck G, Rubio-Texeira M, Shothorst J, Thevelein JM. 2014. Specific analogues uncouple transport, signalling, oligo-ubiquitination and endocytosis in the yeast Gap1 amino acid transceptor. Mol Microbiol 93:213233.

91. Risinger AL, Cain NE, Chen EJ, Kaiser CA. 2006. Activity-dependent reversible inactivation of the general amino acid permease. Mol Biol Cell 17:4411-4419.

92. Donaton MC, Holsbeeks I, Lagatie O, Van Zeebroeck G, Crauwels M, Winderickx J, Thevelein JM. 2003. The Gap1 general amino acid permease acts as an amino acid sensor for activation of protein kinase $A$ targets in the yeast Saccharomyces cerevisiae. Mol Microbiol 50:911-929.

93. Van Zeebroeck G, Bonini BM, Versele M, Thevelein JM. 2009. Transport and signaling via the amino acid binding site of the yeast Gap1 amino acid transceptor. Nat Chem Biol 5:45-52.

94. Crauwels M, Donaton MC, Pernambuco MB, Winderickx J, de Winde JH, Thevelein JM. 1997. The Sch9 protein kinase in the yeast Saccharomyces cerevisiae controls CAPK activity and is required for nitrogen activation of the fermentable-growth-medium-induced (FGM) pathway. Microbiology 143:26272637.

95. Voordeckers K, Kimpe M, Haesendonckx S, Louwet W, Versele M, Thevelein JM. 2011. Yeast 3-phosphoinositide-dependent protein kinase-1 (PDK1) orthologs Pkh1-3 differentially regulate phosphorylation of protein kinase A (PKA) and the protein kinase B (PKB)/S6K ortholog Sch9. J Biol Chem 286:22017-22027.

96. Hirimburegama K, Durnez P, Keleman J, Oris E, Vergauwen R, Mergelsberg H, Thevelein JM. 1992. Nutrient-induced activation of trehalase in nutrientstarved cells of the yeast Saccharomyces cerevisiae: cAMP is not involved as second messenger. J Gen Microbiol 138:2035-2043.

97. Kraidlova L, Van Zeebroeck G, Van Dijck P, Sychrova H. 2011. The Candida albicans GAP gene family encodes permeases involved in general and specific aminio-acid uptake and sensing. Eukaryot Cell 10:1219-1229.

98. Marini AM, Soussi-Boudekou S, Vissers S, André B. 1997. A family of ammonium transporters in Saccharomyces cerevisiae. Mol Biol Cell 17:42824293.

99. Dubois E, Grenson M. 1979. Methylamine/ammonia uptake systems in Saccharomyces cerevisiae: multiplicity and regulation. Mol Gen Genet 175:6776. 
100. Lorenz MC, Heitman J. 1998. The MEP2 ammonium permease regulates pseudohyphal differentiation in Saccharomyces cerevisiae. EMBO J 17:12361247.

101. Biswas K, Morschhauser J. 2005. The Mep2 ammonium permease controls nitrogen starvation-induced filamentous growth in Candida albicans. Mol Microbiol 56:649-669.

102. Smith DG, Garcia-Pedrajas MD, Gold SE, Perlin MH. 2003. Isolation and characterization from pathogenic fungi of genes encoding ammonium permeases and their roles in dimorphism. Mol Microbiol 50:259-275.

103. Rutherford JC, Lin X, Nielsen K, Heitman J. 2008. Amt2 permease is required to induce ammonium-responsive invasive growth and mating in Cryptococcus neoformans. Eukaryot Cell 7:237-246.

104. Mitsuzawa H. 2006. Ammonium transporter genes in the fission yeast Schizosaccharomyces pombe: rol of ammonium uptake and a morphological transition. Genes Cells 11:1183-1195.

105. Van Nuland A, Vandormael P, Donaton M, Alenquer M, Lourenço E, Versele M, Thevelein JM. 2006. Ammonium permease-based sensing mechanism for rapid ammonium activation of the protein kinase A pathway in yeast. Mol Microbiol 59:1485-1505.

106. Cardenas ME, Cutler NS, Lorenz MC, Di Como CJ, Heitman J. 1999. The TOR signaling cascade regulates gene expression in response to nutrients. Genes Dev 13:3271-3279.

107. Boeckstaens M, André B, Marini AM. 2007. The yeast ammonium transport protein Mep2 and its positive regulator, the Npr1 kinase, play an important role in normal and pseudohyphal growth on various nitrogen media through retrieval of excreted ammonium. Mol Microbiol 64:534-546.

108. van den Berg B, Chembath A, Jefferies D, Basle A, Khalid S, Rutherford JC. 2016. Structural basis for Mep2 ammonium transceptor activation by phosphorylation. Nat Commun 7:11337.

109. Khademi S, O'Connell III J, Remis J, Robles-Colmenares Y, Miercke LJW, Stroud RM. 2004. Mechanism of ammonia transport by Amt/MEP/Rh: structure of AmtB at $1.35 \AA$. Science 305:1587-1591.

110. Andrade SL, Dickmanns A, Ficner R, Einsle O. 2005. Crystal structure of the archaeal ammonium transporter Amt-1 from Archaeoglobus fulgidus. Proc Natl Acad Sci U S A 102:14994-14999. 


\section{Zheng L, Kostrewa D, Berneche S, Winkler FK, Li XD. 2004. The}

mechanism of ammonia transport based on the crystal structure of AmtB of Escherichia coli. Proc Natl Acad Sci U S A 101:17090-17095.

\section{Gruswitz F, Chaudhary S, Ho JD, Schlessinger A, Pezeshki B, Ho CM,} Sali A, Westhoff CM, Stroud RM. 2010. Function of human Rh based on structure of RhCG at 2.1 A. Proc Natl Acad Sci U S A 107:9638-9643.

113. Lin Y, Cao Z, Mo Y. 2006. Molecular dynamics simulations on the Escherichia coli ammonia channel protein AmtB: mechanism of ammonia/ammonium transport. J Am Chem Soc 128:10876-10884.

114. Nygaard TP, Rovira C, Peters GH, M.Ø. J. 2006. Ammonium recruitment and ammonia transport by E. coli ammonia channel AmtB. Biophys J 91:44014412.

115. Lamoureux G, Javelle A, Baday S, Wang S, Bernèche S. 2010. Transport mechanisms in the ammonium transporter family. Transfus Clin Biol 17:168-175.

116. Wang S, Orabi EA, Baday S, Bernèche S, Lamoureux G. 2012.

Ammonium transporters achieve charge transfer by fragmenting their substrate. J Am Chem Soc 134:10419-10427.

117. Boeckstaens M, André B, Marini AM. 2008. Distinct transport mechanisms in yeast ammonium transport/sensor proteins of the Mep/Amt/Rh family and impact on filamentation. J Biol Chem 283:21362-21370.

118. Rutherford JC, Chua G, Hughes T, Cardenas ME, Heitman J. 2008. A Mep2-dependent transcriptional profile links permease function to gene expression during pseudohyphal growth in Saccharomyces cerevisiae. Mol Biol Cell 19:3028-3039.

119. Paul JA, Barati MT, Cooper M, Perlin MH. 2014. Physical and genetic interaction between ammonium transporters and the signaling protein Rho1 in the plant pathogen Ustilago Maydis. Eukaryot Cell 13:1328-1336.

120. Teichert S, Rutherford JC, Wottawa M, Heitman J, Tudzynski B. 2008. Impact of ammonium permeases mepA, mepB, and mepC on nitrogen-regulated secondary metabolism in Fusarium fujikuroi. Eukaryot Cell 7:187-201.

121. Javelle A, Morel M, Roriguez-Pastrana BR, Botton B, André B, Marini AM, Brun A, Chalot M. 2003. Molecular characterization, function and regulation of ammonium transporters (Amt) and ammonium-metabolizing enzymes (GS, NADP-GDH) in the ectomycorrhizal fungus Hebeloma cylindrosporum. Mol Microbiol 47:411-430.

122. Shnaiderman C, Miyara I, Kobiler I, Sherman A, Prusky D. 2013.

Differential activation of ammonium transporters during the accumulation of 
ammonia by Colletotrichum gloeosporioides and its effect on appressoria formation and pathogenicity. Mol Plant Microbe Interact 26:345-355.

123. Li L, Wright SJ, Krystofova S, Park G, Borkovich KA. 2007. Heterotrimeric $\mathrm{G}$ protein signaling in filamentous fungi. Annu Rev Microbiol 61:423-452.

124. Thevelein JM, de Winde JH. 1999. Novel sensing mechanisms and targets for the CAMP - protein kinase A pathway in the yeast Saccharomyces cerevisiae. Mol Microbiol 32:1002-1012.

125. Lemaire K, Van De Velde S, Van Dijck P, Thevelein JM. 2004. Glucose and sucrose act as agonist and mannose as antagonist ligands of the $G$ proteincoupled receptor Gpr1 in the yeast Saccharomyces cerevisiae. Mol Cell 16:293299.

126. Yun C-W, Tamaki H, Nakayama R, Yamamoto K, Kumagai H. 1998.

Gpr1p, a putative G-protein coupled receptor, regulates glucose-dependent cellular cAMP level in yeast Saccharomyces cerevisiae. Biochem Biophys Res Commun 252:29-33.

127. Rolland F, de Winde JH, Lemaire K, Boles E, Thevelein JM, Winderickx J. 2000. Glucose-induced cAMP signalling in yeast requires both a G-protein coupled receptor system for extracellular glucose detection and a separable hexose kinase-dependent sensing process. Mol Microbiol 38:348-358.

128. Deschenes R-J, Broach J-R. 1989. The function of RAS genes in Saccharomyces cerevisiae. Adv Cancer Res 54:79-138.

129. Colombo S, Ma P, Cauwenberg L, Winderickx J, Crauwels M, Teunissen A, Nauwelaers D, de Winde JH, Gorwa MF, Colavizza D, Thevelein JM. 1998. Involvement of distinct G-proteins, Gpa2 and Ras, in glucose- and intracellular acidification-induced cAMP signalling in the yeast Saccharomyces cerevisiae. EMBO J 17:3326-3341.

130. Versele M, de Winde JH, Thevelein JM. 1999. A novel regulator of Gprotein signalling in yeast, Rgs2, downregulates glucose-activation of the cAMP pathway through direct inhibition of Gpa2. EMBO J 18:5577-5591.

131. Lorenz MC, Pan X, Harashima T, Cardenas ME, Xue Y, Hirsch JP, Heitman J. 2000. The G protein-coupled receptor Gpr1 is a nutrient sensor that regulates pseudohyphal differentiation in Saccharomyces cerevisiae. Genetics 154:609-622.

132. Johnson C, Kweon HK, Sheidy D, Shively CA, Mellacheruvu D, Nesvizhskii Al, Andrews PC, Kumar A. 2014. The yeast Sks1p kinase signaling network regulates pseudohyphal growth and glucose response. PloS Genetics 10:e1004183. 
133. Harashima T, Heitman J. 2002. The Galpha protein Gpa2 controls yeast differentiation by interacting with Kelch repeat proteins that mimic $G$ beta subunits. Mol Cell 10:163-173.

134. Niranjan T, Guo X, Victor J, Lu A, Hirsch JP. 2007. Kelch repeat protein interacts with the yeast Galpha subunit Gpa2p at a site that couples receptor binding to guanine nucleotide exchange. J Biol Chem 282:24231-24238.

135. Zeller CE, Parnell SC, DohIman HG. 2007. The RACK1 ortholog Asc1 functions as a G-protein beta subunit coupled to glucose responsiveness in yeast. J Biol Chem 282:25168-25176.

136. Peeters T, Louwet W, Gelade R, Nauwelaers D, Thevelein JM, Versele M. 2006. Kelch-repeat proteins interacting with the Galpha protein $\mathrm{Gpa} 2$ bypass adenylate cyclase for direct regulation of protein kinase $A$ in yeast. Proc Natl Acad Sci USA 103:13034-13039.

137. Budhwar R, Fang G, Hirsch JP. 2011. Kelch repeat proteins control yeast PKA activity in response to nutrient availability. Cell Cycle 10:767-770.

138. Budhwar R, Lu A, Hirsch JP. 2010. Nutrient control of yeast PKA activity involves opposing effects on phosphorylation of the Bcy1 regulatory subunit. Mol Biol Cell 21:3749-3758.

139. Willhite DG, Brigati JR, Selcer KE, Denny JE, Duck ZA, Wright SE. 2014. Pheromone responsiveness is regulated by components of the Gpr1p-mediated glucose sensing pathway in Saccharomyces cerevisiae. Yeast 31:361-374.

140. Van Dijck P. 2009. Nutrient sensing G protein-coupled receptors: interesting targets for antifungals. Med Mycol 47:671-680.

141. Maidan MM, De Rop L, Serneels J, Exler S, Rupp S, Tournu H, Thevelein JM, Van Dijck P. 2005. The G protein-coupled receptor Gpr1 and the Galpha protein Gpa2 act through the cAMP-protein kinase A pathway to induce morphogenesis in Candida albicans. Mol Biol Cell 16:1971-1986.

142. Miwa T, Takagi Y, Shinozaki M, Yun C-W, Schell WA, Perfect JR, Kumagai H, Tamaki H. 2004. Gpr1, a putative G-protein-coupled receptor, regulates morphogenesis and hypha formation in the pathogenic fungus Candida albicans. Eukaryot Cell 3:919-931.

143. Maidan MM, Thevelein JM, Van Dijck P. 2005. Carbon source induced yeast-to-hypha transition in Candida albicans is dependent on the presence of amino acids and on the G-protein-coupled receptor Gpr1. Biochem Soc Trans 33:291-293.

144. Kozubowski L, Lee SC, Heitman J. 2009. Signalling pathways in the pathogenesis of Cryptococcus. Cell Microbiol 11:370-380. 
145. Xue C, Bahn YS, Cox GM, Heitman J. 2006. G protein-coupled receptor Gpr4 senses amino acids and activates the cAMP-PKA pathway in Cryptococcus neoformans. Mol Biol Cell 17:667-679.

146. Okagaki LH, Wang Y, Ballou ER, O'Meara TR, Bahn YS, Alspaugh JA, Xue C, Nielsen K. 2011. Cryptococcal titan cell formation is regulated by Gprotein signaling in response to multiple stimuli. Eukaryot Cell 10:1306-1316.

147. Li LD, Borkovich KA. 2006. GPR-4 is a predicted G-protein-coupled receptor required for carbon source-dependent asexual growth and development in Neurospora crassa. Eukaryot Cell 5:1287-1300.

148. de Souza WR, Morais ER, Krohn NG, Savoldi M, Goldman MHS, Rodrigues F, Caldana C, Semelka CT, Tikunov AP, Macdonald JM, Goldman GH. 2013. Identification of Metabolic Pathways Influenced by the GProtein Coupled in Aspergillus nidulans. Plos One 8:e62088.

149. Han KH, Seo JA, Yu JH. 2004. A putative G protein-coupled receptor negatively controls sexual development in Aspergillus nidulans. Mol Microbiol 51:1333-1345.

150. Brown NA, dos Reis TF, Ries LNA, Caldana C, Mah J-H, Yu J-H, Macdonald JM, Goldman GH. 2015. G-protein coupled receptor-mediated nutrient sensing and developmental control in Aspergillus nidulans. Mol Microbiol 98:420-439.

151. Gehrke A, Heinekamp T, Jacobsen ID, Brakhage AA. 2010. Heptahelical Receptors GprC and GprD of Aspergillus fumigatus Are Essential Regulators of Colony Growth, Hyphal Morphogenesis, and Virulence. Appl Environ Microbiol 76:3989-3998.

152. Affeldt KJ, Carrig J, Amare M, Keller NP. 2014. Global Survey of Canonical Aspergillus flavus G Protein-Coupled Receptors. mBio 5:e01501-14.

153. Krohn NG, Brown NA, Colabardini AC, Reis T, Savoldi M, Dinamarco TM, Goldman MHS, Goldman GH. 2014. The Aspergillus nidulans ATM Kinase Regulates Mitochondrial Function, Glucose Uptake and the Carbon Starvation Response. G3 (Bethesda) 4:49-62.

154. Krystofova S, Borkovich KA. 2006. The predicted G-protein-coupled receptor GPR-1 is required for female sexual development in the multicellular fungus Neurospora crassa. Eukaryot Cell 5:1503-1516.

155. Chung KS, Won M, Lee SB, Jang YJ, Hoe KL, Kim DU, Lee JW, Kim KW, Yoo HS. 2001. Isolation of a novel gene from Schizosaccharomyces pombe: stm1(+) encoding a seven-transmembrane loop protein that may couple with the heterotrimeric G alpha 2 protein, Gpa2. J Biol Chem 276:40190-40201. 
156. Rispail N, Soanes DM, Ant C, Czajkowski R, Grunler A, Huguet R, PerezNadales E, Poli A, Sartorel E, Valiante V, Yang M, Beffa R, Brakhage AA, Gow NAR, Kahmann R, Lebrun M-H, Lenasi H, Perez-Martin J, Talbot NJ, Wendland J, Di Pietro A. 2009. Comparative genomics of MAP kinase and calcium-calcineurin signalling components in plant and human pathogenic fungi. Fungal Genet Biol 46:287-298.

157. Turra D, El Ghalid M, Rossi F, Di Pietro A. 2015. Fungal pathogen uses sex pheromone receptor for chemotropic sensing of host plant signals. Nature 527:521-524.

158. DeZwaan TM, Carroll AM, Valent B, Sweigard JA. 1999. Magnaporthe grisea Pth $11 \mathrm{p}$ is a novel plasma membrane protein that mediates appressorium differentiation in response to inductive substrate cues. Plant Cell 11:2013-2030.

159. Ramanujam R, Calvert ME, Selvaraj P, Naqvi NI. 2013. The Late Endosomal HOPS Complex Anchors Active G-Protein Signaling Essential for Pathogenesis in Magnaporthe oryzae. PloS Pathog 9:e1003527.

160. Kulkarni RD, Thon MR, Pan HQ, Dean RA. 2005. Novel G-protein-coupled receptor-like proteins in the plant pathogenic fungus Magnaporthe grisea. Genome Biol 6.R24.

161. Cabrera IE, Pacentine IV, Lim A, Guerrero N, Krystofova S, Li L, Michkov AV, Servin JA, Ahrendt SR, Carrillo AJ, Davidson LM, Barsoum AH, Cao J, Castillo R, Chen W-C, Dinkchian A, Kim S, Kitada SM, Lai TH, Mach A, Malekyan C, Moua TR, Torres CR, Yamamoto A, Borkovich KA. 2015. Global Analysis of Predicted G Protein-Coupled Receptor Genes in the Filamentous Fungus, Neurospora crassa. G3 (Bethesda) 5:2729-2743.

162. Cullen PJ, Sabbagh W, Graham E, Irick MM, van Olden EK, Neal C, Delrow J, Bardwell L, Sprague GF. 2004. A signaling mucin at the head of the Cdc42- and MAPK-dependent filamentous growth pathway in yeast. Genes Dev 18:1695-1708.

163. Cullen PJ, Sprague GF. 2000. Glucose depletion causes haploid invasive growth in yeast. Proc Natl Acad Sci USA 97:13619-13624.

164. Karunanithi S, Cullen PJ. 2012. The Filamentous Growth MAPK Pathway Responds to Glucose Starvation Through the Mig1/2 Transcriptional Repressors in Saccharomyces cerevisiae. Genetics 192:869-887.

165. Karunanithi S, Joshi J, Chavel C, Birkaya B, Grell L, Cullen PJ. 2012. Regulation of Mat Responses by a Differentiation MAPK Pathway in Saccharomyces cerevisiae. PLoS One 7:e32294 
166. Cullen PJ. 2007. Signaling mucins: The new kids on the MAPK block. Crit Rev Eukaryot Gene Expr 17:241-257.

167. Vadaie N, Dionne H, Akajagbor DS, Nickerson SR, Krysan DJ, Cullen PJ. 2008. Cleavage of the signaling mucin Msb2 by the aspartyl protease Yps1 is required for MAPK activation in yeast. J Cell Biol 181:1073-1081.

168. Verstrepen KJ, Klis FM. 2006. Flocculation, adhesion and biofilm formation in yeasts. Mol Microbiol 60:5-15.

169. Guo B, Styles CA, Feng QH, Fink GR. 2000. A Saccharomyces gene family involved in invasive growth, cell-cell adhesion, and mating. Proceedings of the National Academy of Sciences of the United States of America 97:12158-12163.

170. Finkel JS, Mitchell AP. 2011. Genetic control of Candida albicans biofilm development. Nat Rev Microbiol 9:109-118.

171. Roman E, Cottier F, Ernst JF, Pla J. 2009. Msb2 Signaling Mucin Controls Activation of Cek1 Mitogen-Activated Protein Kinase in Candida albicans.

Eukaryot Cell 8:1235-1249.

172. Puri S, Kumar R, Chadha S, Tati S, Conti HR, Hube B, Cullen PJ, Edgerton M. 2012. Secreted Aspartic Protease Cleavage of Candida albicans Msb2 Activates Cek1 MAPK Signaling Affecting Biofilm Formation and Oropharyngeal Candidiasis. PLoS One 7:e46020.

173. Szafranski-Schneider E, Swidergall M, Cottier F, Tielker D, Roman E, Pla J, Ernst JF. 2012. Msb2 Shedding Protects Candida albicans against Antimicrobial Peptides. Plos Pathogens 8:e1002501.

174. Swidergall M, Ernst AM, Ernst JF. 2013. Candida albicans Mucin Msb2 Is a Broad-Range Protectant against Antimicrobial Peptides. Antimicrob Agents Chemother 57:3917-3922.

175. Brown NA, dos Reis TF, Goinski AB, Savoldi M, Menino J, Almeida MT, Rodrigues F, Goldman GH. 2014. The Aspergillus nidulans signalling mucin MsbA regulates starvation responses, adhesion and affects cellulase secretion in response to environmental cues. Mol Microbiol 94:1103-1120.

176. Perez-Nadales E, Di Pietro A. 2011. The Membrane Mucin Msb2 Regulates Invasive Growth and Plant Infection in Fusarium oxysporum. Plant Cell 23:11711185.

177. Perez-Nadales E, Di Pietro A. 2015. The transmembrane protein Sho1 cooperates with the mucin Msb2 to regulate invasive growth and plant infection in Fusarium oxysporum. Mol Plant Pathol 16:593-603. 


\section{Lanver D, Berndt P, Tollot M, Naik V, Vranes M, Warmann T, Muench K,}

Roessel N, Kahmann R. 2014. Plant Surface Cues Prime Ustilago maydis for Biotrophic Development. PLoS Pathog 10:e1004272.

179. Lanver D, Mendoza-Mendoza A, Brachmann A, Kahmann R. 2010. Sho1 and Msb2-Related Proteins Regulate Appressorium Development in the Smut Fungus Ustilago maydis. Plant Cell 22:2085-2101.

180. Liu W, Zhou X, Li G, Li L, Kong L, Wang C, Zhang H, Xu J-R. 2011. Multiple Plant Surface Signals are Sensed by Different Mechanisms in the Rice Blast Fungus for Appressorium Formation. Plos Pathog 7:e1001261.

181. Wang G, Li G, Zhang S, Jiang C, Qin J, Xu J-R. 2015. Activation of the signalling mucin MoMsb2 and its functional relationship with Cbp1 in Magnaporthe oryzae. Environm Microbiol 17:2969-2981.

182. Kamakura T, Yamaguchi S, Saitoh K, Teraoka T, Yamaguchi I. 2002. A novel gene, CBP1, encoding a putative extracellular chitin-binding protein, may play an important role in the hydrophobic surface sensing of Magnaporthe grisea during appressorium differentiation. Mol Plant Microbe Interact 15:437-444.

\section{Figure legends}

Figure 1

Sugar sensing proteins in the plasma membrane of fungal cells. Nutrient sensing proteins in general can be devided into non-transporting receptors, transceptors and G-protein coupled receptors (GPCRs). Non-transporting sugar receptors include the Saccharomyces cerevisiae (Sc) glucose sensors Snf3/Rgt2, the Kluyveromyces lactis $(K I)$ glucose sensor Rag4, the Hansenula polymorpha $(H p)$ glucose sensor $\mathrm{Hxs} 1$, the Candida albicans ( $\mathrm{Ca}$ ) glucose sensor Hgt4, the Cryptococcus neoformans $(\mathrm{Cn})$ inositol sensor Itr1, the Trichoderma reesei $(\mathrm{Tr})$ cellobiose sensor Crt1and the Cn cellodextrin sensor Clp1. Sugar transceptors include the Ca glucose sensor Hgt12, the Cn hexose sensors Hxs1/2, the Hp hexose sensor Gcr1, the Neurospora crassa $(\mathrm{Nc})$ sugar sensor Rco3, the Collectrichum graminicola $(\mathrm{Cg})$ hexose sensor $\mathrm{Hxt4}$, the Ustilago maydis $(\mathrm{Um})$ hexose sensor Hxt1, the Nc cellobiose sensors Cdt1/2 and the Trichoderma reesei $(T C)$ cellobiose sensor Stp1. Sugar GPCRs include the Sc glucose sensor Gpr1, the Cn glucose sensors Gpr4/5, the Nc glucose sensor Gpr4, the Aspergillus nidulans (An) glucose sensors $\mathrm{GprD} / \mathrm{H}$, the Aspergillus fumigatus ( $A f$ ) glucose sensors GprC/D and the Aspergillus flavus ( $A f$ ) glucose sensors GprA/C/J/K/R. 


\section{Figure 2}

Nitrogen sensing proteins in the plasma membrane of fungal cells. Nutrient sensing proteins in general can be devided into non-transporting receptors, transceptors and G-protein coupled receptors (GPCRs). Non-transporting nitrogen receptors include the Saccharomyces cerevisiae (Sc) amino acid sensor Ssy1, the Candida albicans (Ca) amino acid sensor Csy1 and an unknown Cryptococcus neoformans (Cn) amino acid sensor. Nitrogen transceptors include the $S c$ amino acid sensor Gap1, the $\mathrm{Ca}$ amino acid sensors Gap1/2/6, the Sc ammonium sensor Mep2, the Ca ammonium sensor Mep2, the Ustilago maydis (Um) ammonium sensor Ump2, the Fusarium fujikuroi $(F f)$ ammonium sensors MepA-C, the Hebeloma cylindrosporum (Hc) ammonium sensors Amt1-3, the Tuber borchii (Tb) ammonium sensor Amt1 and the Colletotrichum gloeosporoiodes $(\mathrm{Cg})$ ammonium sensors MepA-C. Nitrogen GPCRs include the $\mathrm{Ca}$ methionine sensor Gpr1, the $C$ ) amino acid sensor Gpr4, the Aspergillus nidulans ( $A n$ ) tryptophan sensor $\mathrm{GprH}$, the Aspergillus flavus (Af) proline sensor GprC/D, the Schyzosaccharomyces pombe (Sp) nitrogen sensor Stm1 and the Af ammonium sensor GprR.

\section{Figure 3}

Surface sensing proteins in the plasma membrane of fungal cells. Characterized surface sensing proteins can be divided into G-protein coupled receptors (GPCRs) and the interacting Sho receptors and the extracellular mucin receptors. The nonclassical, Pth11-type, GPCRs include the Magnaporthe oryzae (Mo) Pth11 receptor of hydrophobic surfaces and the Neurospora crassa (Nc) Gpr32, Gpr36 and Gpr39 putative lignocellulose receptors. The Sho receptors include the orthologous Ustilago maydis (Um), Fusarium oxysporum (Fo) and Mo, hydrophobic surface Sho1 receptors. The extracellular mucins include the orthologous Aspergillus nidulans (An), Um, Fo, Mo, Msb2 hydrophobic surface receptors, in addition to the Msb2 receptors of Candida albicans ( $\mathrm{Ca}$ ) and Saccharomyces cerevisiae (Sc) that have not been characterized as detecting surfaces, plus the additional Cbp1 mucin receptor of hydrophobic surfaces from Mo.

\section{Figure 4}

Nutrient and surface sensing proteins in the plasma membrane of fungal cells and the downstream signal transduction pathways they trigger. Examples for the different classes of nutrient (non-transporting receptors, transceptors and GPCRs) and surface (Mucin and Sho) sensing proteins are shown. Sensing of nutrients by nontransporting receptors and sugar transceptors results in the induction of nutrient 
transporter genes. Nitrogen transceptors (e.g. ScGap1) result in the activation of the PKA pathway upon sensing appropriate nitrogen sources. Depending on the type of GPCR, they can activate the CAMP-PKA pathway, the MAPK pathway or both. The surface receptors activate the MAPK pathway. 
Fig 1. glucose.pdf $107 / 11 / 16 \quad 17: 27$

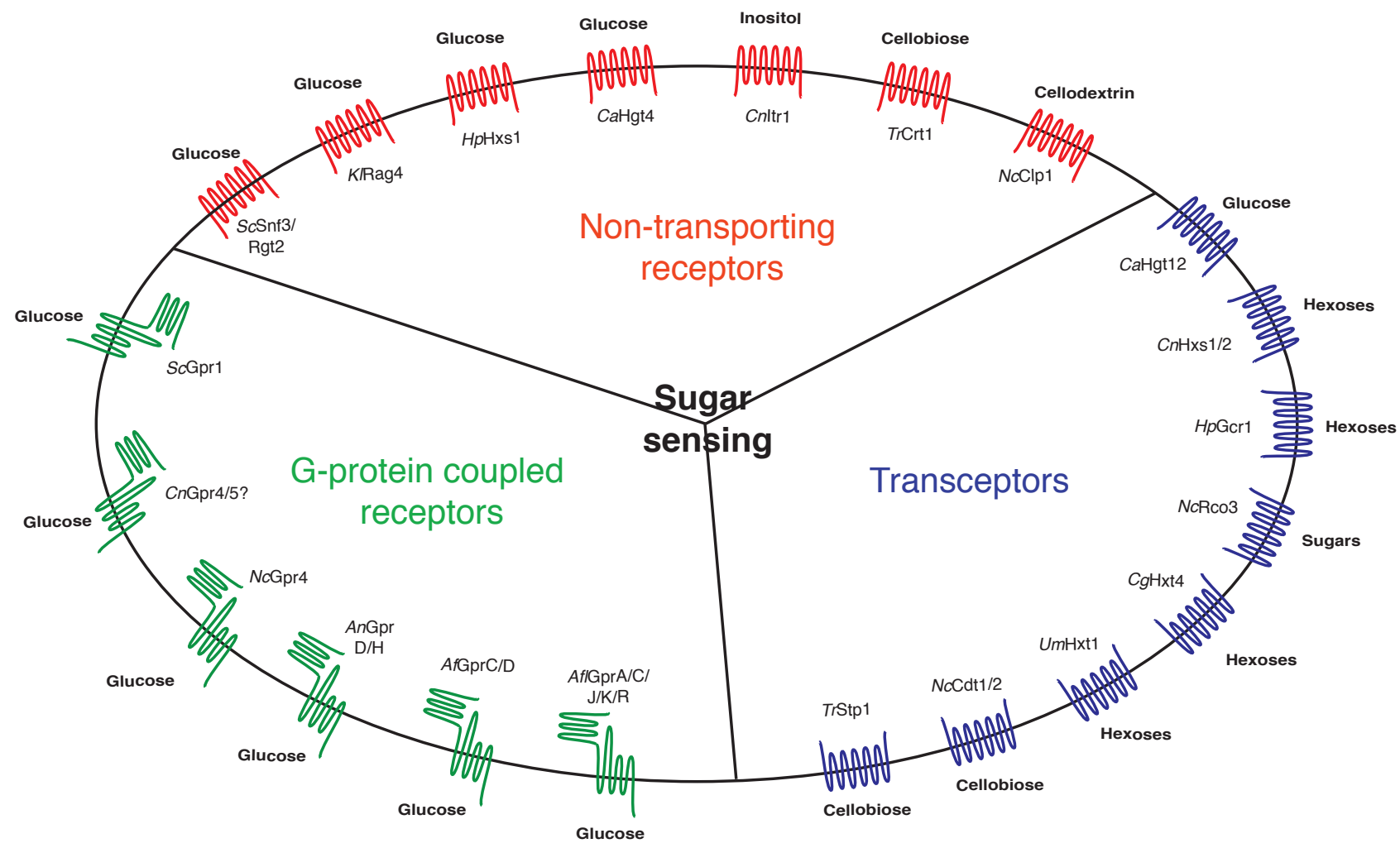




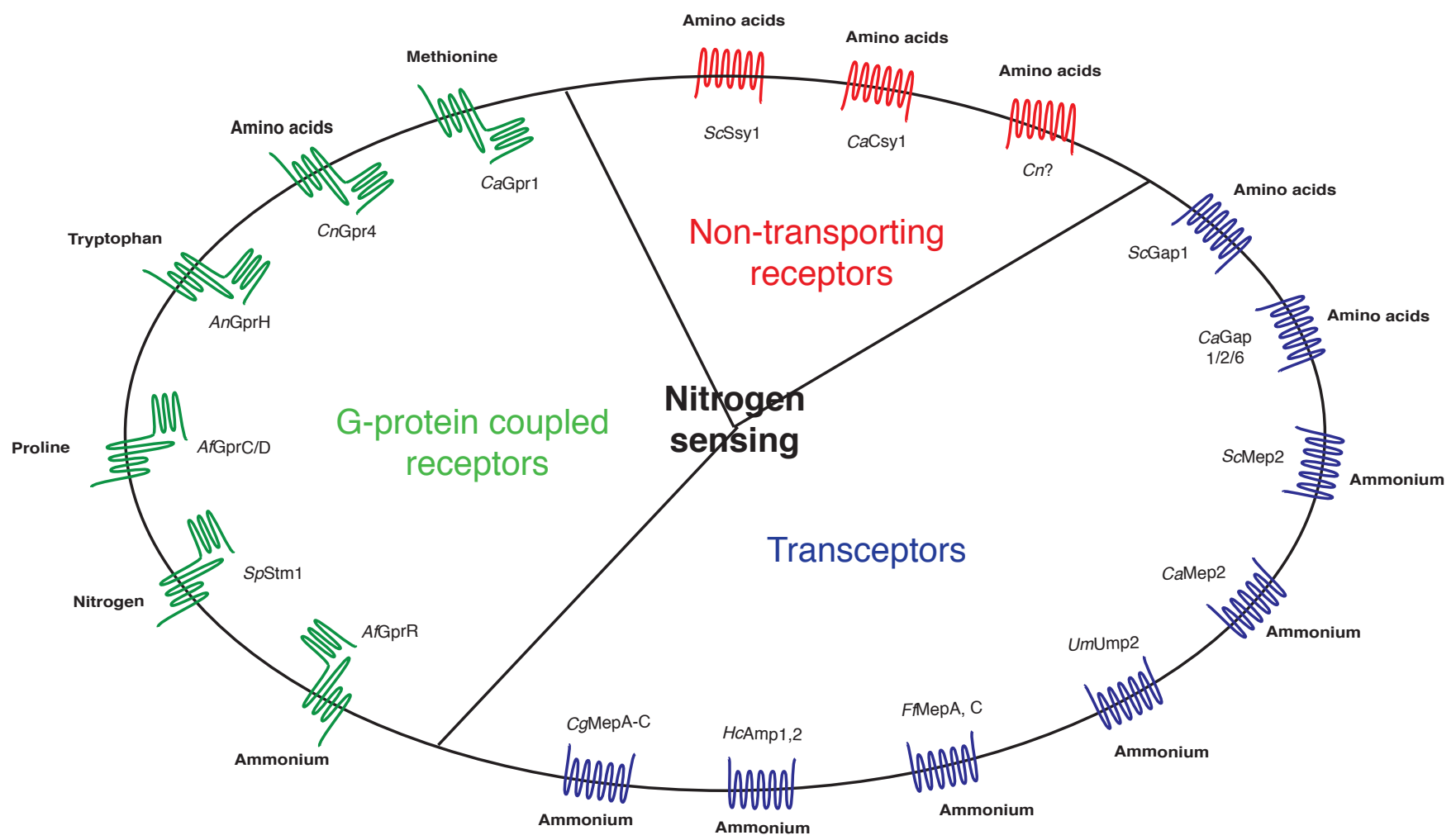




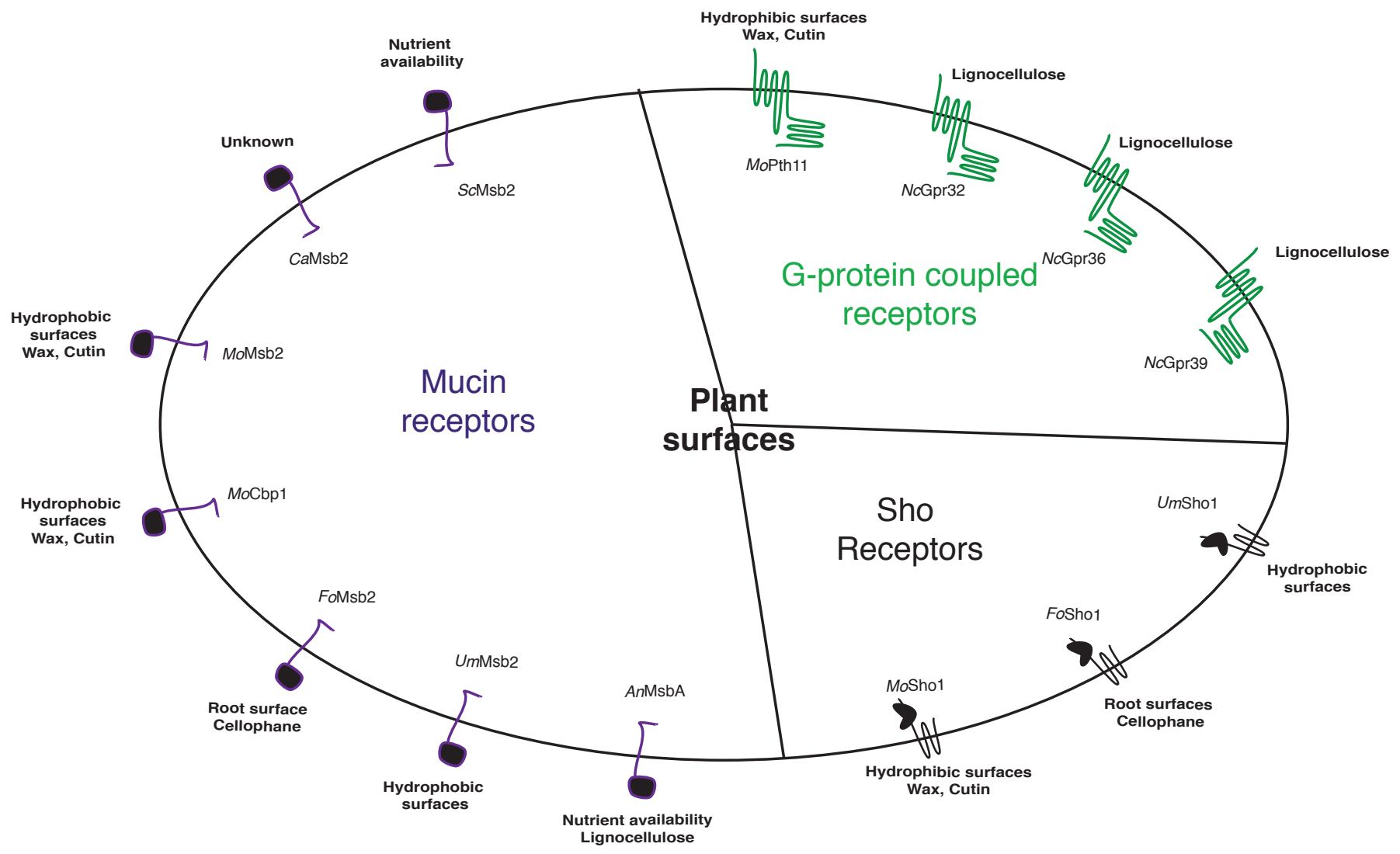




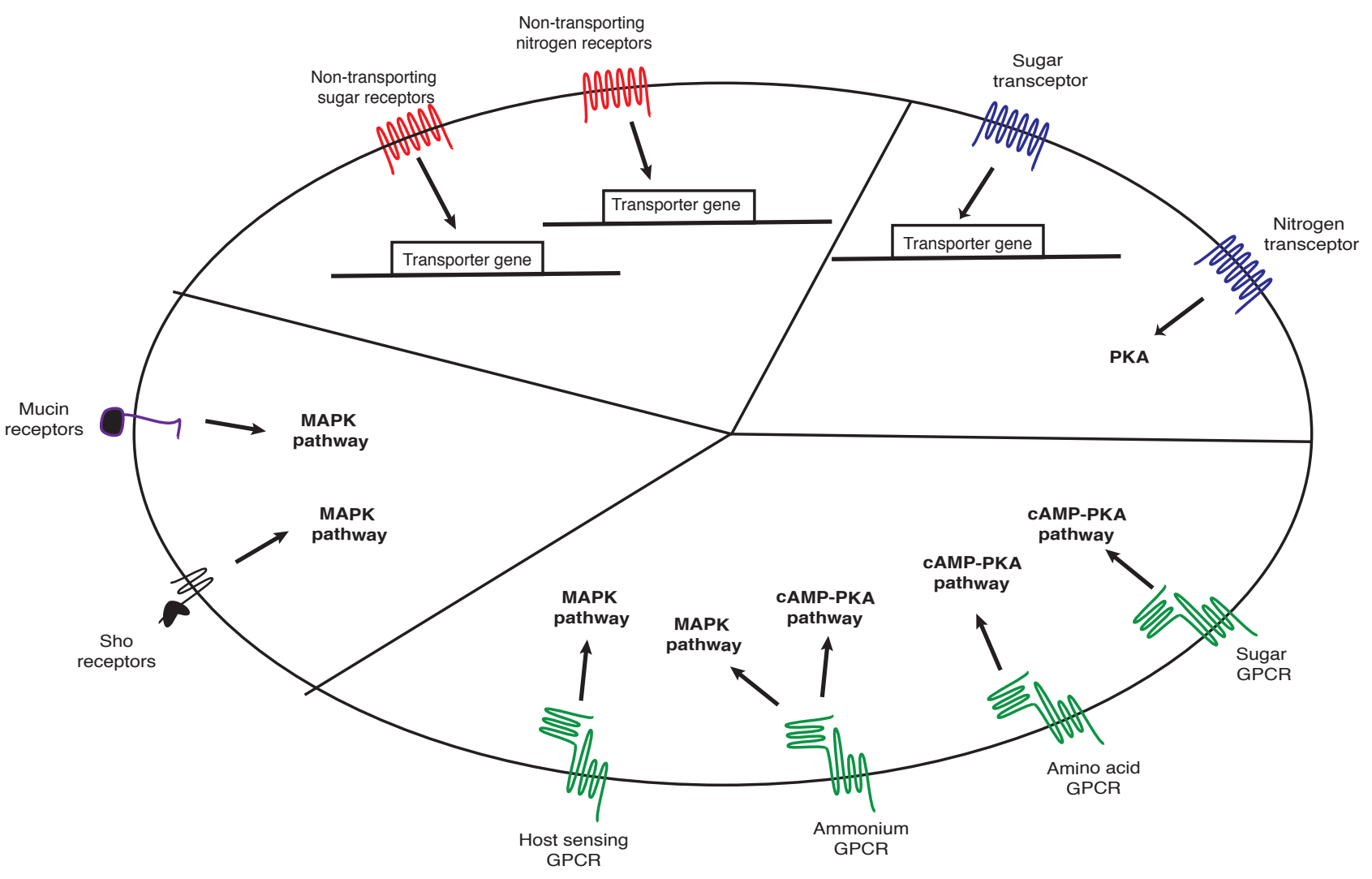

